\title{
A MULTI-SITE CAMPAIGN TO MEASURE SOLAR-LIKE OSCILLATIONS IN PROCYON. II. MODE FREQUENCIES
}

\author{
Timothy R. Bedding ${ }^{1}$, Hans Kueldsen ${ }^{2}$, Tiago L. Campante ${ }^{2,3}$, Thierry Appourchaux ${ }^{4}$, Alfio Bonanno ${ }^{5}$, \\ William J. Chaplin ${ }^{6}$, Rafael A. Garcia ${ }^{7}$, Milena Martić ${ }^{8}$, Benoit Mosser ${ }^{9}$, R. Paul Butler ${ }^{10}$, Hans Bruntt $^{1,11}$, \\ László L. Kiss ${ }^{1,12}$, Simon J. O’Toole ${ }^{13}$, Eiji Kambe ${ }^{14}$, Hiroyasu Ando ${ }^{15}$, Hideyuki Izumiura ${ }^{14}$, Bun'ei Sato ${ }^{16}$, \\ Michael Hartmann ${ }^{17}$, Artie Hatzes ${ }^{17}$, Caroline Barban ${ }^{11}$, Gabrielle Berthomieu ${ }^{18}$, Eric Michel ${ }^{11}$, Janine Provost $^{18}$, \\ Sylvaine Turck-Chièze ${ }^{7}$, Jean-Claude Lebrun ${ }^{8}$, Jerome Schmitt $^{19}{ }^{19}$ Jean-Loup Bertaux $^{8}$, Serena Benatti ${ }^{20}$, \\ Riccardo U. Claudi ${ }^{21}$, Rosario Cosentino ${ }^{5}$, Silvio Leccia ${ }^{22}$, Søren Frandsen ${ }^{2}$, Karsten Brogatad ${ }^{2}$, Lars Glowienka ${ }^{2}$, \\ Frank Grundahl ${ }^{2}$, Eric Stempels ${ }^{23}$, Torben Arentoft ${ }^{2}$, Michaël Bazot $^{2}$, Jørgen Christensen-DalsgaArD ${ }^{2}$, \\ Thomas H. Dall ${ }^{24}$, Christoffer KarofF ${ }^{2}$, Jens Lundgreen-Nielsen ${ }^{2}$, Fabien Carrier $^{25}$, Patrick Eggenberger ${ }^{26}$, \\ Danuta Sosnowska ${ }^{27}$, Robert A. Wittenmyer ${ }^{28,29}$, Michael Endl ${ }^{28}$, Travis S. Metcalfe ${ }^{30}$, Saskia HeKker $^{6,31}$, \\ AND SABINE REFFERT ${ }^{32}$ \\ ${ }^{1}$ Sydney Institute for Astronomy (SIfA), School of Physics, University of Sydney, NSW 2006, Australia; bedding @ physics.usyd.edu.au \\ ${ }^{2}$ Danish AsteroSeismology Centre (DASC), Department of Physics and Astronomy, Aarhus University, DK-8000 Aarhus C, Denmark \\ ${ }^{3}$ Centro de Astrofísica da Universidade do Porto, Rua das Estrelas, 4150-762 Porto, Portugal \\ ${ }^{4}$ Institut d'Astrophysique Spatiale, Université Paris XI-CNRS, Bâtiment 121, 91405 Orsay Cedex, France \\ 5 INAF-Osservatorio Astrofisico di Catania, via S. Sofia 78, 95123 Catania, Italy \\ ${ }^{6}$ School of Physics and Astronomy, University of Birmingham, Edgbaston, Birmingham B15 2TT, UK \\ ${ }^{7}$ DAPNIA/DSM/Service d'Astrophysique, CEA/Saclay, 91191 Gif-sur-Yvette Cedex, France \\ ${ }^{8}$ LATMOS, University of Versailles St Quentin, CNRS, BP 3, 91371 Verrieres le Buisson Cedex, France \\ ${ }^{9}$ LESIA, CNRS, Université Pierre et Marie Curie, Université Denis Diderot, Observatoire de Paris, 92195 Meudon Cedex, France \\ ${ }^{10}$ Carnegie Institution of Washington, Department of Terrestrial Magnetism, 5241 Broad Branch Road NW, Washington, DC 20015-1305, USA \\ ${ }^{11}$ Laboratoire AIM, CEA/DSM-CNRS-Université Paris Diderot-IRFU/SAp, 91191 Gif-sur-Yvette Cedex, France \\ ${ }^{12}$ Konkoly Observatory of the Hungarian Academy of Sciences, H-1525 Budapest, P.O. Box 67, Hungary \\ ${ }_{13}$ Anglo-Australian Observatory, P.O. Box 296, Epping, NSW 1710, Australia \\ ${ }^{14}$ Okayama Astrophysical Observatory, National Astronomical Observatory of Japan, National Institutes of Natural Sciences, 3037-5 Honjyo, Kamogata, Asakuchi, \\ Okayama 719-0232, Japan \\ ${ }^{15}$ National Astronomical Observatory of Japan, National Institutes of Natural Sciences, 2-21-1 Osawa, Mitaka, Tokyo 181-8588, Japan \\ ${ }^{16}$ Global Edge Institute, Tokyo Institute of Technology 2-12-1-S6-6, Ookayama, Meguro-ku, Tokyo 152-8550, Japan \\ ${ }^{17}$ Thüringer Landessternwarte Tautenburg, Sternwarte 5, 07778 Tautenburg, Germany \\ ${ }^{18}$ Université de Nice Sophia-Antipolis, CNRS UMR 6202, Laboratoire Cassiopée, Observatoire de la Côte d'Azur, BP 4229, 06304 Nice Cedex, France \\ ${ }^{19}$ Observatoire de Haute Provence, 04870 St Michel l'Observatoire, France \\ ${ }^{20}$ CISAS-University of Padova, Via Venezia 5, 35131, Padova, Italy \\ ${ }^{21}$ INAF-Astronomical Observatory of Padua, Vicolo Osservatorio 5, 35122 Padova, Italy \\ 22 INAF-Osservatorio Astronomico di Capodimonte, Salita Moiariello 16, 80131 Napoli, Italy \\ ${ }^{23}$ Department of Physics and Astronomy, P.O. Box 516, SE-751 20 Uppsala, Sweden \\ ${ }^{24}$ European Southern Observatory, D-85748 Garching, Germany \\ ${ }^{25}$ Instituut voor Sterrenkunde, Katholieke Universiteit Leuven, Celestijnenlaan 200 B, 3001 Leuven, Belgium \\ ${ }^{26}$ Observatoire de Genève, Université de Genève, Ch. des Maillettes 51, CH-1290 Sauverny, Switzerland \\ ${ }^{27}$ Laboratoire d'astrophysique, EPFL Observatoire CH-1290 Versoix, Switzerland \\ ${ }^{28}$ McDonald Observatory, University of Texas at Austin, Austin, TX 78712, USA \\ ${ }^{29}$ School of Physics, University of New South Wales, NSW 2052, Australia \\ ${ }^{30}$ High Altitude Observatory, National Centre for Atmospheric Research, Boulder, CO 80307-3000, USA \\ ${ }^{31}$ Leiden Observatory, Leiden University, 2300 RA Leiden, The Netherlands \\ ${ }^{32}$ ZAH-Landessternwarte, 69117 Heidelberg, Germany \\ Received 2010 January 19; accepted 2010 February 26; published 2010 March 29
}

\begin{abstract}
We have analyzed data from a multi-site campaign to observe oscillations in the F5 star Procyon. The data consist of high-precision velocities that we obtained over more than three weeks with 11 telescopes. A new method for adjusting the data weights allows us to suppress the sidelobes in the power spectrum. Stacking the power spectrum in a so-called échelle diagram reveals two clear ridges, which we identify with even and odd values of the angular degree $(l=0$ and 2 , and $l=1$ and 3 , respectively). We interpret a strong, narrow peak at $446 \mu \mathrm{Hz}$ that lies close to the $l=1$ ridge as a mode with mixed character. We show that the frequencies of the ridge centroids and their separations are useful diagnostics for asteroseismology. In particular, variations in the large separation appear to indicate a glitch in the sound-speed profile at an acoustic depth of $\sim 1000 \mathrm{~s}$. We list frequencies for 55 modes extracted from the data spanning 20 radial orders, a range comparable to the best solar data, which will provide valuable constraints for theoretical models. A preliminary comparison with published models shows that the offset between observed and calculated frequencies for the radial modes is very different for Procyon than for the Sun and other cool stars. We find the mean lifetime of the modes in Procyon to be $1.29_{-0.49}^{+0.55}$ days, which is significantly shorter than the $2-4$ days seen in the Sun.
\end{abstract}

Key words: stars: individual (Procyon A) - stars: oscillations 


\section{INTRODUCTION}

The success of helioseismology and the promise of asteroseismology have motivated numerous efforts to measure oscillations in solar-type stars. These began with ground-based observations (for recent reviews see Bedding \& Kjeldsen 2007; Aerts et al. 2008) and now extend to space-based photometry, particularly with the CoRoT and Kepler Missions (e.g., Michel et al. 2008; Gilliland et al. 2010).

We have carried out a multi-site spectroscopic campaign to measure oscillations in the F5 star Procyon A (HR 2943; HD 61421; HIP 37279). We obtained high-precision velocity observations over more than three weeks with 11 telescopes, with almost continuous coverage for the central 10 days. In Arentoft et al. (2008, hereafter Paper I) we described the details of the observations and data reduction, measured the mean oscillation amplitudes, gave a crude estimate for the mode lifetime and discussed slow variations in the velocity curve that we attributed to rotational modulation of active regions. In this paper, we describe the procedure used to extract the mode parameters, provide a list of oscillation frequencies, and give an improved estimate of the mode lifetimes.

\section{PROPERTIES OF SOLAR-LIKE OSCILLATIONS}

We begin with a brief summary of the relevant properties of solar-like oscillations (for reviews see, for example, Brown \& Gilliland 1994; Bedding \& Kjeldsen 2003; ChristensenDalsgaard 2004).

To a good approximation, in main-sequence stars the cyclic frequencies of low-degree $p$-mode oscillations are regularly spaced, following the asymptotic relation (Tassoul 1980; Gough 1986):

$$
v_{n, l} \approx \Delta v\left(n+\frac{1}{2} l+\epsilon\right)-l(l+1) D_{0} .
$$

Here, $n$ (the radial order) and $l$ (the angular degree) are integers, $\Delta v$ (the large separation) depends on the sound travel time across the whole star, $D_{0}$ is sensitive to the sound speed near the core and $\epsilon$ is sensitive to the reflection properties of the surface layers. It is conventional to define three so-called small frequency separations that are sensitive to the sound speed in the core: $\delta v_{02}$ is the spacing between adjacent modes with $l=0$ and $l=2$ (for which $n$ will differ by 1 ); $\delta v_{13}$ is the spacing between adjacent modes with $l=1$ and $l=3$ (ditto); and $\delta v_{01}$ is the amount by which $l=1$ modes are offset from the midpoint of the $l=0$ modes on either side. ${ }^{33}$ To be explicit, for a given radial order, $n$, these separations are defined as follows:

$$
\begin{gathered}
\delta v_{02}=v_{n, 0}-v_{n-1,2}, \\
\delta v_{01}=\frac{1}{2}\left(v_{n, 0}+v_{n+1,0}\right)-v_{n, 1}, \\
\delta v_{13}=v_{n, 1}-v_{n-1,3} .
\end{gathered}
$$

If the asymptotic relation (Equation (1)) were to hold exactly, it would follow that all of these separations would be independent of $n$ and that $\delta v_{02}=6 D_{0}, \delta v_{13}=10 D_{0}$, and $\delta v_{01}=2 D_{0}$. In practice, Equation (1) is only an approximation. In the Sun and other stars, theoretical models and observations show that

\footnotetext{
33 One can also define an equivalent quantity, $\delta v_{10}$, as the offset of $l=0$ modes from the midpoint between the surrounding $l=1$ modes, so that $\delta v_{10}=v_{n, 0}-\frac{1}{2}\left(v_{n-1,1}+v_{n, 1}\right)$.
}

$\Delta v, D_{0}$, and $\epsilon$ vary somewhat with frequency, and also with $l$. Consequently, the small separations also vary with frequency.

The mode amplitudes are determined by the excitation and damping, which are stochastic processes involving nearsurface convection. We typically observe modes over a range of frequencies, which in Procyon is especially broad (about 400-1400 $\mu \mathrm{Hz}$; see Paper I). The observed amplitudes also depend on $l$ via various projection factors (see Table 1 of Kjeldsen et al. 2008a). Note, in particular, that velocity measurements are much more sensitive to modes with $l=3$ than are intensity measurements. The mean mode amplitudes are modified for a given observing run by the stochastic nature of the excitation, resulting in considerable scatter of the peak heights about the envelope.

Oscillations in the Sun are long-lived compared to their periods, which allows their frequencies to be measured very precisely. However, the lifetime is not infinite and the damping results in each mode in the power spectrum being split into multiple peaks under a Lorentzian profile. The FWHM of this Lorentzian, which is referred to as the linewidth $\Gamma$, is inversely proportional to the mode lifetime: $\Gamma=1 /(\pi \tau)$. We follow the usual definition that $\tau$ is the time for the mode amplitude to decay by a factor of $e$. The solar value of $\tau$ for the strongest modes ranges from 2 to 4 days, as a decreasing function of frequency (e.g., Chaplin et al. 1997).

Procyon is an evolved star, with theoretical models showing that it is close to, or just past, the end of the main sequence (e.g., Guenther \& Demarque 1993; Barban et al. 1999; Chaboyer et al. 1999; Di Mauro \& Christensen-Dalsgaard 2001; Kervella et al. 2004; Eggenberger et al. 2005; Provost et al. 2006; Bonanno et al. 2007; Guenther et al. 2008). As such, its oscillation spectrum may show deviations from the regular comb-like structure described by Equation (1), especially at low frequencies. This is because some modes, particularly those with $l=1$, are shifted by avoided crossings with gravity modes in the stellar core (also called "mode bumping"; see Osaki 1975; Aizenman et al. 1977). These so-called "mixed modes" have $p$-mode character near the surface but $g$-mode character in the deep interior. Some of the theoretical models of Procyon cited above indeed predict these mixed modes, depending on the evolutionary state of the star, and we must keep this in mind when attempting to identify oscillation modes in the power spectrum. The mixed modes are rich in information because they probe the stellar core and are very sensitive to age, but they complicate the task of mode identification.

We should also keep in mind that mixed modes are expected to have lower amplitudes and longer lifetimes (smaller linewidths) than regular $p$-modes because they have larger mode inertias (e.g., Christensen-Dalsgaard 2004). Hence, for a data series that is many times longer than the lifetime of the pure $p$-modes, a mixed mode may appear in the power spectrum as a narrow peak that is higher than the others, even though its power (amplitude squared) is not especially large.

Another potential complication is that stellar rotation causes modes with $l \geqslant 1$ to split into multiplets. The peaks of these multiplets are characterized by the azimuthal degree $m$, which takes on values of $m=0, \pm 1, \ldots, \pm l$, with a separation that directly measures the rotation rate averaged over the region of the star that is sampled by the mode. The measurements are particularly difficult because a long time series is needed to resolve the rotational splittings. We argue in Appendix A that the low value of $v \sin i$ observed in Procyon implies that rotational splitting of frequencies is not measurable in our observations. 

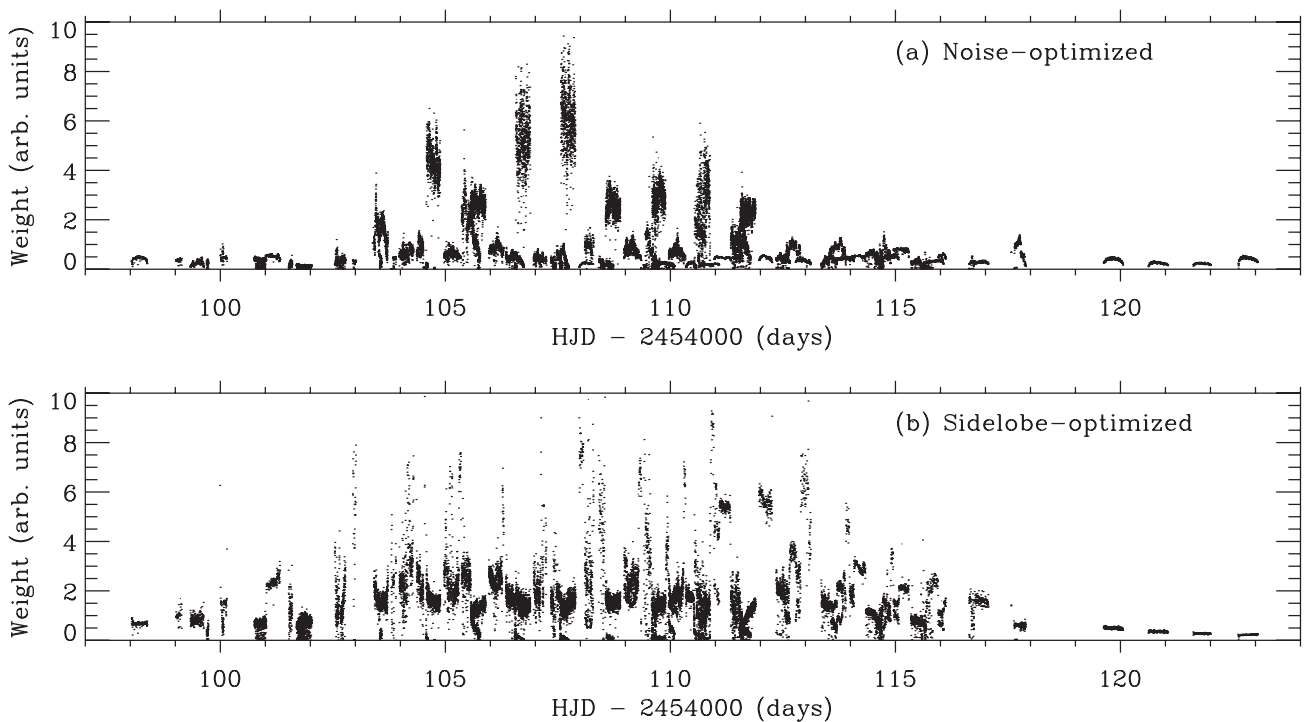

Figure 1. Weights for time series of velocity observations of Procyon, optimized to minimize: (a) the noise level and (b) the height of the sidelobes.

\section{WEIGHTING THE TIME SERIES}

The time series of velocity observations was obtained over 25 days using 11 telescopes at eight observatories and contains just over 22,500 data points. As discussed in Paper I, the velocity curve shows slow variations that we attribute to a combination of instrumental drifts and rotational modulation of stellar active regions. We have removed these slow variations by subtracting all the power below $280 \mu \mathrm{Hz}$, to prevent spectral leakage into higher frequencies that would degrade the oscillation spectrum. We take this high-pass-filtered time series of velocities, together with their associated measurement uncertainties, as the starting point in our analysis.

\subsection{Noise-optimized Weights}

Using weights when analyzing ground-based observations of stellar oscillations (e.g., Gilliland et al. 1993; Frandsen et al. 1995) allows one to take into account the significant variations in data quality during a typical observing campaign, especially when two or more telescopes are used. The usual practice, which we followed in Paper I, is to calculate the weights for a time series from the measurement uncertainties, $\sigma_{i}$, according to $w_{i}=1 / \sigma_{i}^{2}$.

These "raw" weights can then be adjusted to minimize the noise level in the final power spectrum by identifying and revising those uncertainties that are too optimistic, and at the same time rescaling the uncertainties to be in agreement with the actual noise levels in the data. This procedure is described in Paper I and references therein. The time series of these noiseoptimized weights is shown in Figure 1(a). These are the same as those shown in Figure 1(d) of Paper I, but this time as weights rather than uncertainties.

The power spectrum of Procyon based on these noiseoptimized weights is shown in Figure 2(a). This is the same as shown in Paper I (lower panel of Figure 6), except that the power at low frequencies, which arises from the slow variations, has been removed. As described in Paper I, the noise level above $3 \mathrm{mHz}$ in this noise-optimized spectrum is $1.9 \mathrm{~cm} \mathrm{~s}^{-1}$ in amplitude. This includes some degree of spectral leakage from the oscillations and if we high-pass filter the spectrum up to $3 \mathrm{mHz}$ to remove the oscillation signal, the noise level drops to $1.5 \mathrm{~cm} \mathrm{~s}^{-1}$ in amplitude.
The task of extracting oscillation frequencies from the power spectrum is complicated by the presence of structure in the spectral window, which are caused by gaps or otherwise uneven coverage in the time series. The spectral window using the noise-optimized weights is shown in Figure 3(a). Prominent sidelobes at $\pm 11.57 \mu \mathrm{Hz}$ correspond to aliasing at one cycle per day. Indeed, the prospect of reducing these sidelobes is the main reason for acquiring multi-site observations. However, even with good coverage the velocity precision varies greatly, both for a given telescope during the run and from one telescope to another (see Figure 1(a)). As pointed out in Paper I, using these measurement uncertainties as weights has the effect of increasing the sidelobes in the spectral window. We now discuss a technique for addressing this issue.

\subsection{Sidelobe-optimized Weights}

Adjusting the weights allows one to suppress the sidelobe structure; the trade-off is an increase in the noise level. This technique is routinely used in radio astronomy when synthesizing images from interferometers (e.g., Högbom \& Brouw 1974). An extreme case is to set all weights to be equal, which is the same as not using weights at all. This is certainly not optimal because it produces a power spectrum with greatly increased noise (by a factor of 2.3) but still having significant sidelobes, as can be seen in Figure 6(a) of Paper I. Adjusting the weights on a night-by-night basis in order to minimize the sidelobes was used in the analysis of dual-site observations of $\alpha$ Cen A (Bedding et al. 2004), $\alpha$ Cen B (Kjeldsen et al. 2005), and $\beta$ Hyi (Bedding et al. 2007). For our multi-site Procyon data, this is impractical because of the large number of (partly overlapping) telescope nights. We have developed a more general algorithm for adjusting weights to minimize the sidelobes $(\mathrm{H}$. Kjeldsen et al. 2010, in preparation). The new method, which is superior because it does not assume the oscillations are coherent over the whole observing run, is based on the principle that equal weight is given to all segments of the time series. The method produces the cleanest possible spectral window in terms of suppressing the sidelobes, and we have tested it with good results using published data for $\alpha$ Cen A and B and $\beta$ Hyi (Arentoft et al. 2010).

The new method operates with two timescales, $T_{1}$ and $T_{2}$. All data segments of length $T_{1}(=2 \mathrm{hr}$, in this case) are required to 

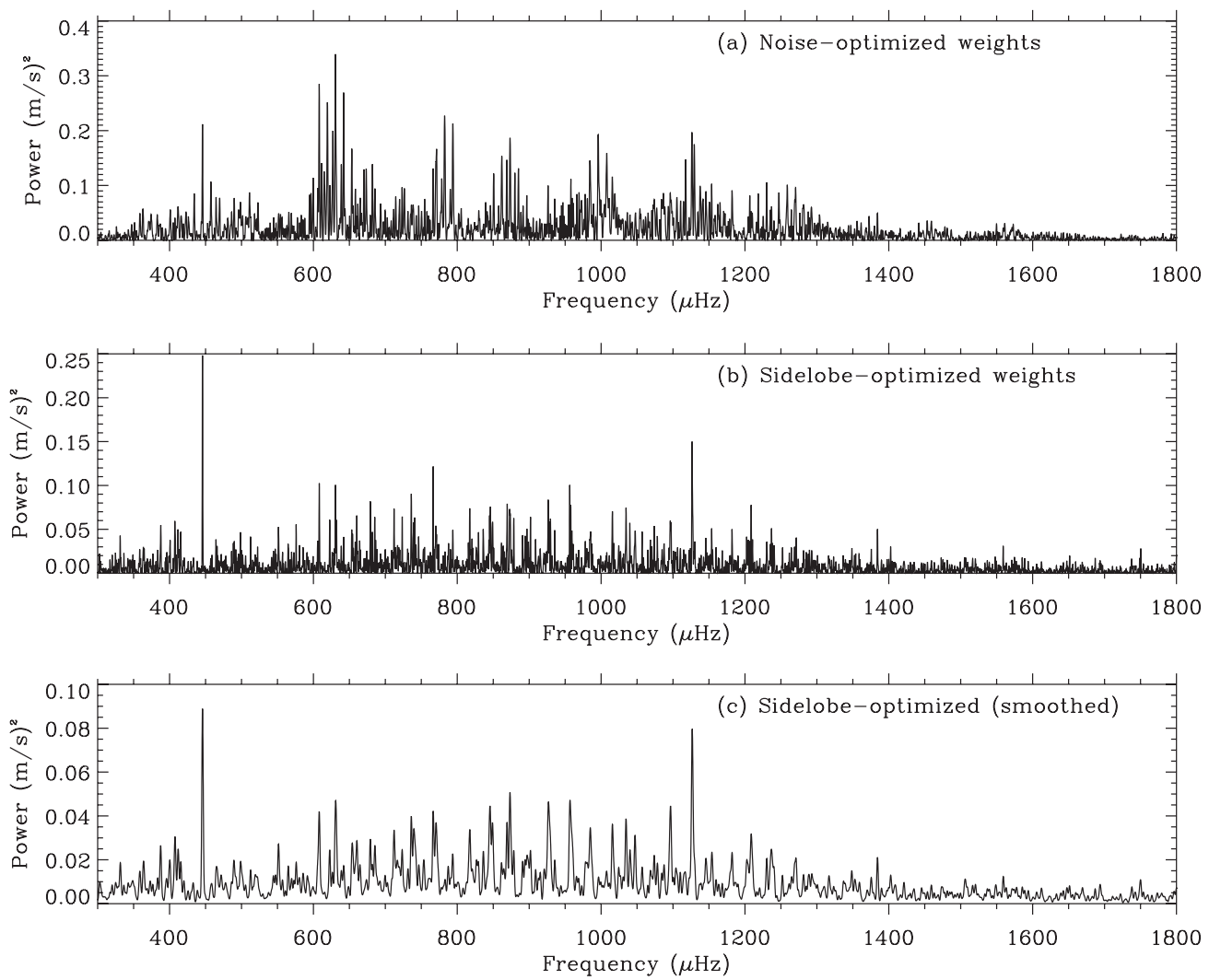

Figure 2. Power spectrum of oscillations in Procyon: (a) using the noise-optimized weights; (b) using the sidelobe-optimized weights; (c) using the sidelobe-optimized weights and smoothing by convolution with a Gaussian with FWHM $2 \mu \mathrm{Hz}$.
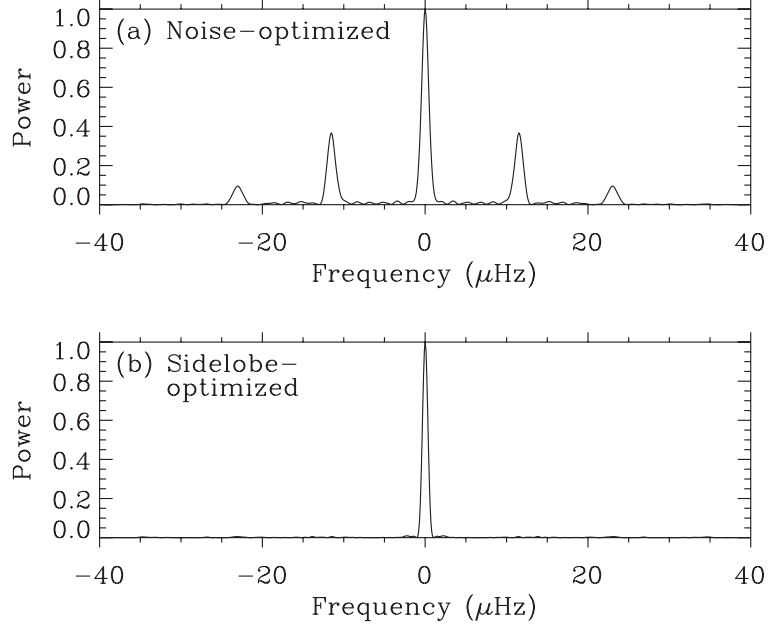

Figure 3. Spectral window for the Procyon observations using (a) noiseoptimized weights and (b) sidelobe-optimized weights.

have the same total weight throughout the time series, with the relaxing condition that variations on timescales longer than $T_{2}$ $(=12 \mathrm{hr})$ are retained. To be explicit, the algorithm works as follows. We adjust the weights so that all segments of length $T_{1}$ have the same total weight. That is, for each point $w_{i}$ in the time series of weights, define $\left\{S_{i}\right\}$ to be the set of weights in a segment of width $T_{1}$ centered at that time stamp, and divide each $w_{i}$ by the sum of the weights in $\left\{S_{i}\right\}$. However, this adjustment suffers from edge effects, since it gives undue weight to points adjacent to a gap. To compensate, we also divide by an asymmetry factor

$$
R=1+\left|\frac{\Sigma_{\text {left }}-\Sigma_{\text {right }}}{\Sigma_{\text {left }}+\Sigma_{\text {right }}}\right| .
$$

Here, $\Sigma_{\text {left }}$ is the sum of the weights in the segment $\left\{S_{i}\right\}$ that have time stamps less than $t_{i}$, and $\Sigma_{\text {right }}$ is the sum of the weights in the segment $\left\{S_{i}\right\}$ that have time stamps greater than $t_{i}$. Note that $R$ ranges from 1 , for points that are symmetrically placed in their $T$ bin, up to 2 for points at one edge of a gap.

Once the above procedure is done for $T_{1}$, which is the shortest timescale on which we wish to adjust the weights, we do it again with $T_{2}$, which is the longest timescale for adjusting the weights. Finally, we divide the first set of adjusted weights by the second set, and this gives the weights that we adopt (Figure 1(b)).

\subsection{The Sidelobe-optimized Power Spectrum}

Figure 2(b) shows the power spectrum of Procyon based on the sidelobe-optimized weights. The spectral window has improved tremendously (Figure 3(b)), while the noise level at high frequencies (above $3 \mathrm{mHz}$ ) has increased by a factor of 2.0 .

The power spectrum now clearly shows a regular series of peaks, which are even more obvious after smoothing (Figure 2(c)). We see that the large separation of the star is about $55 \mu \mathrm{Hz}$, confirming the value indicated by several previous studies (Mosser et al. 1998; Martić et al. 1999, 2004; Eggenberger et al. 2004; Régulo \& Roca Cortés 2005; Leccia et al. 2007; Guenther et al. 2008). The very strong peak at $446 \mu \mathrm{Hz}$ appears to be a candidate for a mixed mode, especially given its narrowness (see Section 2).

Plotting the power spectrum in échelle format using a large separation of $56 \mu \mathrm{Hz}$ (Figure 4) clearly shows two ridges, as 


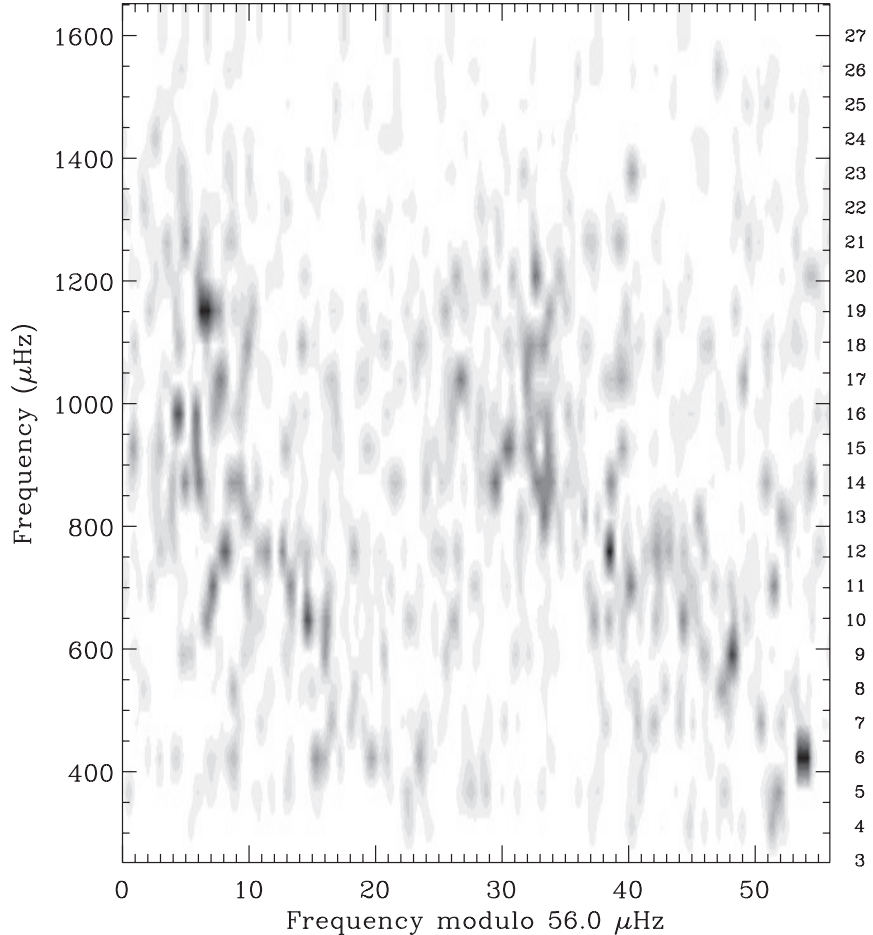

Figure 4. Power spectrum of Procyon in échelle format using a large separation of $56 \mu \mathrm{Hz}$, based on the sidelobe-optimized weights. Two ridges are clearly visible. The upper parts are vertical but the lower parts are tilted, indicating a change in the large separation as a function of frequency. The orders are numbered sequentially on the right-hand side.

expected. $^{34}$ The upper parts are vertical but the lower parts are tilted, indicating a change in the large separation as a function of frequency. This large amount of curvature in the échelle diagram goes a long way toward explaining the lack of agreement between previous studies on the mode frequencies of Procyon (see the list of references given in the previous paragraph).

The advantage of using the sidelobe-optimized weights is demonstrated by Figure 5. This is the same as Figure 4 but for the noise-optimized weights and the ridges are no longer clearly defined.

\section{IDENTIFICATION OF THE RIDGES}

We know from asymptotic theory (see Equation (1)) that one of the ridges in the échelle diagram (Figure 4) corresponds to modes with even degree $(l=0$ and 2$)$ and the other to modes with odd degree ( $l=1$ and 3 ). However, it is not immediately obvious which is which. We also need to keep in mind that the asymptotic relation in evolved stars does not hold exactly. We designate the two possibilities Scenario A, in which the left-hand ridge in Figure 4 corresponds to modes with odd degree, and Scenario B, in which the same ridge corresponds to modes with even degree. Figure 6 shows the Procyon power spectrum collapsed along several orders. We now see double peaks that suggest the identifications shown, which corresponds to Scenario B.

\footnotetext{
34 When making an échelle diagram, it is common to plot $v$ versus $(v \bmod \Delta v)$, in which case each order slopes slightly upward. However, for gray-scale images, it is preferable to keep the orders horizontal, as was done in the original presentation of the diagram (Grec et al. 1983). We have followed that approach in this paper, and the value given on the vertical axis indicates the frequency at the middle of each order.
}

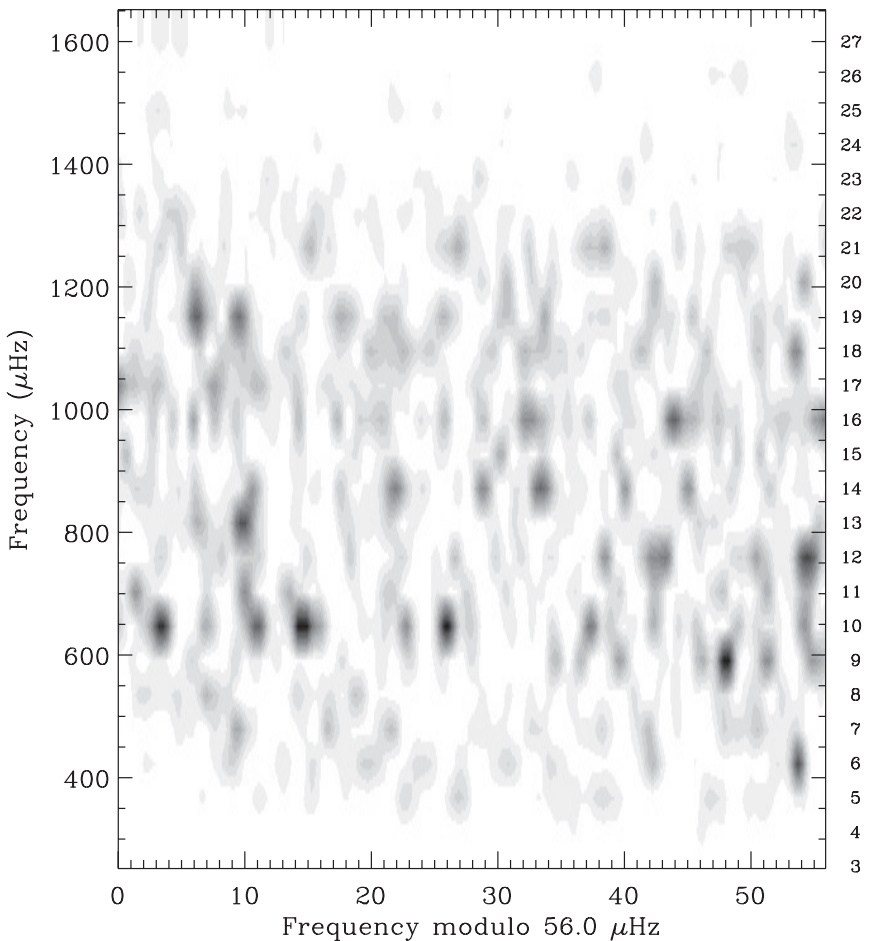

Figure 5. Same as Figure 4, but for the noise-optimized weights. The sidelobes from daily aliasing mean that the ridges can no longer be clearly distinguished.
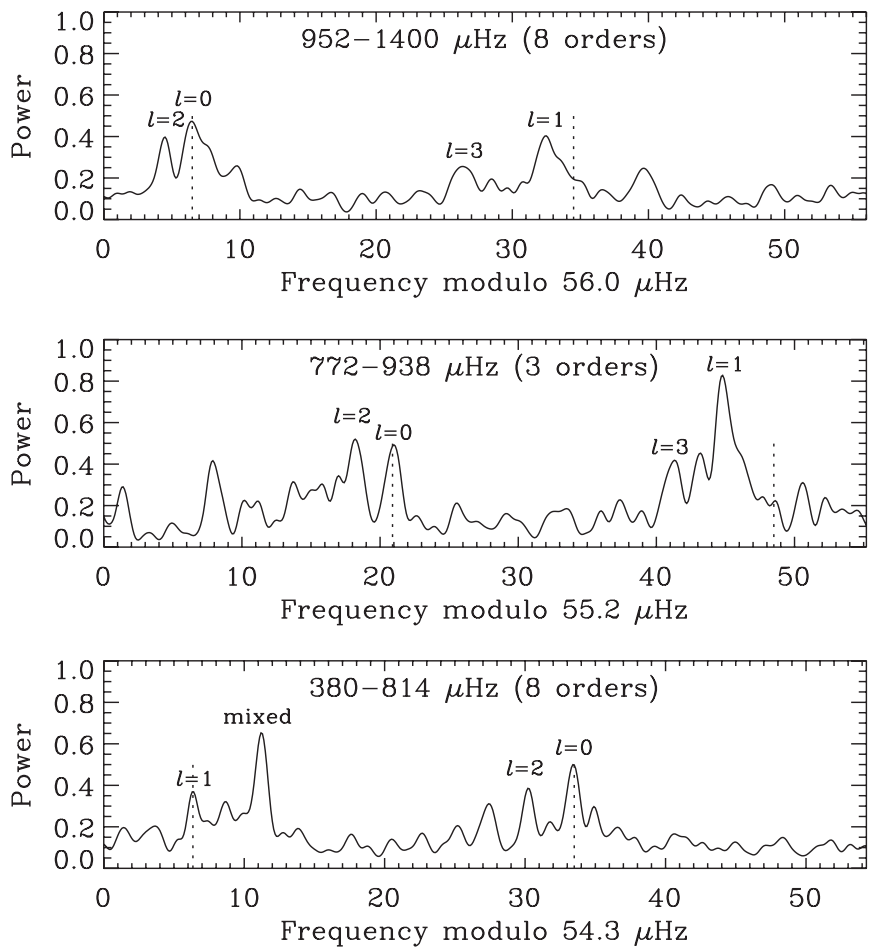

Figure 6. Power spectrum of Procyon collapsed along several orders. Note that the power spectrum was first smoothed slightly by convolving with a Gaussian with FWHM $0.5 \mu \mathrm{Hz}$. The dotted lines are separated by exactly $\Delta \nu / 2$, to guide the eye in assessing the $0-1$ small separation.

We can check that the small separation $\delta v_{01}$ has the expected sign. According to asymptotic theory (Equation (1)), each $l=1$ mode should be at a slightly lower frequency than the midpoint of the adjacent $l=0$ modes. This is indeed the case for the identifications given in Figure 6, but would not be if the even 


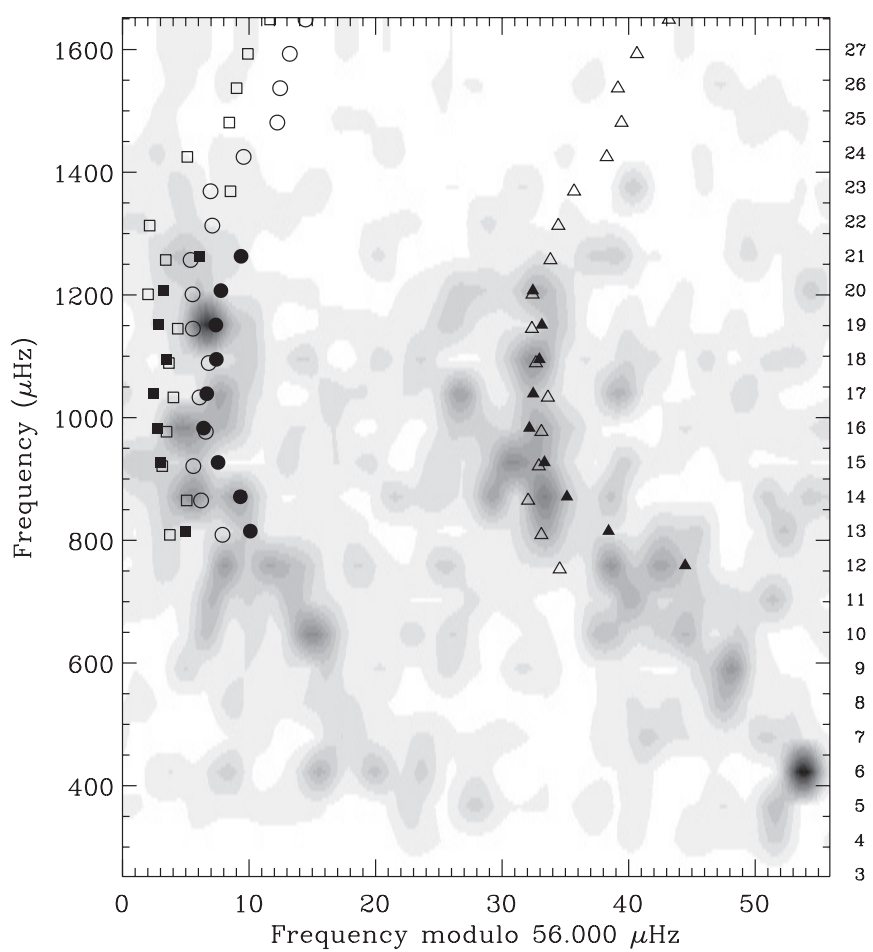

Figure 7. Échelle diagram for Procyon smoothed to $2 \mu \mathrm{Hz}$ (grayscale) overlaid with scaled frequencies for two stars observed by $\mathrm{CoRoT}$. The filled symbols are oscillation frequencies for HD 49385 reported by Deheuvels et al. (2010), after multiplying by 0.993 . Open symbols are oscillation frequencies for HD 49933 from the revised identification by Benomar et al. (2009b, Scenario B) after multiplying by 0.6565 . Symbol shapes indicate mode degree: $l=0$ (circles), $l=1$ (triangles), and $l=2$ (squares).

and odd degrees were reversed. We should be careful, however, since $\delta v_{01}$ has been observed to have the opposite sign in red giant stars (Carrier et al. 2010; Bedding et al. 2010).

The problem of ridge identification in $\mathrm{F}$ stars was first encountered by Appourchaux et al. (2008) when analyzing CoRoT observations of HD 49933 and has been followed up by numerous authors (Benomar et al. 2009a, 2009b; Gruberbauer et al. 2009; Mosser \& Appourchaux 2009; Roxburgh 2009; Kallinger et al. 2010). Two other F stars observed by CoRoT have presented the same problem, namely HD 181906 (García et al. 2009) and HD 181420 (Barban et al. 2009). A discussion of the issue was recently given by Bedding \& Kjeldsen (2010), who proposed a solution to the problem that involves comparing two (or more) stars on a single échelle diagram after first scaling their frequencies.

Figure 7 shows the échelle diagram for Procyon overlaid with scaled frequencies for two stars observed by CoRoT, using the method described by Bedding \& Kjeldsen (2010). The filled symbols are scaled oscillation frequencies for the G0 star HD 49385 observed by CoRoT (Deheuvels et al. 2010). The scaling involved multiplying all frequencies by a factor of 0.993 before plotting them, with this factor being chosen to align the symbols as closely as possible with the Procyon ridges. For this star, the CoRoT data gave an unambiguous mode identification, which is indicated by the symbol shapes. This confirms that the left-hand ridge of Procyon corresponds to modes with even $l$ (Scenario B).

The open symbols in Figure 7 are oscillation frequencies for HD 49933 from the revised identification by Benomar et al. (2009b, Scenario B), after multiplying by a scaling factor of

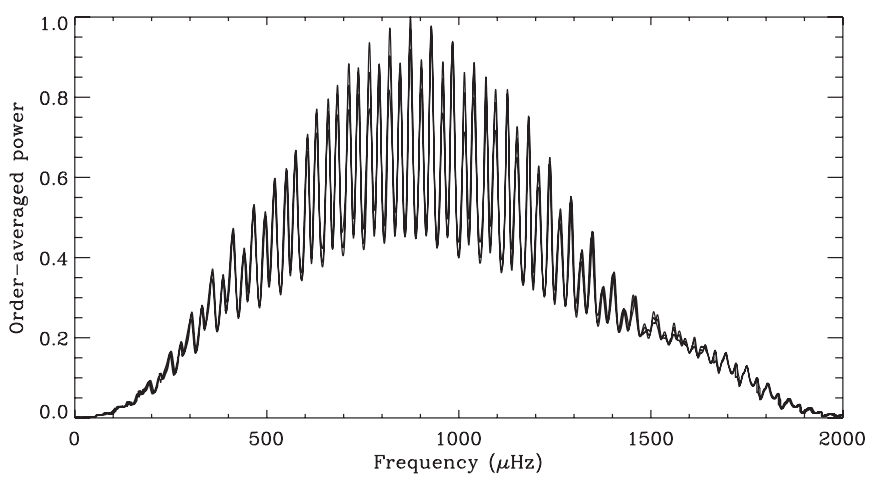

Figure 8. Order-averaged power spectrum (OAPS), where smoothing was done with a FWHM of 4.0 orders (see the text). The OAPS is plotted for three values of the large separations $(54,55$, and $56 \mu \mathrm{Hz})$, and we see that the positions of the maxima are not very sensitive to the value of $\Delta v$.

0.6565. The alignment with HD 49385 was already demonstrated by Bedding \& Kjeldsen (2010). We show HD 49933 here for comparison and to draw attention to the different amounts of bending at the bottom of the right-hand $(l=1)$ ridge for the three stars. The CoRoT target that is most similar to Procyon is HD170987 but unfortunately the signal-to-noise ratio $(\mathrm{S} / \mathrm{N})$ is too low to provide a clear identification of the ridges (Mathur et al. 2010).

The above considerations give us confidence that Scenario B in Procyon is the correct identification, and we now proceed on that basis.

\section{FREQUENCIES OF THE RIDGE CENTROIDS}

Our next step in the analysis was to measure the centroids of the two ridges in the échelle diagram. We first removed the strong peak at $446 \mu \mathrm{Hz}$ (it was replaced by the mean noise level). We believe this to be a mixed mode and its extreme power means that it would significantly distort the result. We then smoothed the power spectrum to a resolution of $10 \mu \mathrm{Hz}$ (FWHM). To further improve the visibility of the ridges, we also averaged across several orders, which corresponds to smoothing in the vertical direction in the échelle diagram (Bedding et al. 2004; Kjeldsen et al. 2005; Karoff 2007). That is, for a given value of $\Delta \nu$, we define the "order-averaged" power spectrum to be

$$
\operatorname{OAPS}(v, \Delta v)=\sum_{j=-4}^{4} c_{j} \operatorname{PS}(v+j \Delta v) .
$$

The coefficients $c_{j}$ are chosen to give a smoothing with a FWHM of $k \Delta v$ :

$$
c_{j}=c_{-j}=\frac{1}{1+(2 j / k)^{2}} .
$$

We show in Figure 8 the OAPS based on smoothing over 4 orders $(k=4.0)$, and so we used $\left(c_{0}, \ldots, c_{4}\right)=(1,0.8,0.5,0.31,0.2)$. The OAPS is plotted for three values of the large separations (54, 55, and $56 \mu \mathrm{Hz}$ ), and they are superimposed. The three curves are hardly distinguishable, and we see that the positions of the maxima are not sensitive to the value of $\Delta v$.

We next calculated a modified version of the OAPS in which the value at each frequency is the maximum value of the OAPS over a range of large separations $(53-57 \mu \mathrm{Hz})$. This is basically the same as the comb response, as used previously by several authors (Kjeldsen et al. 1995; Mosser et al. 1998; Martić et al. 1999; Leccia et al. 2007). The maxima of this function define the centroids of the two ridges, which are shown in Figure 9. 


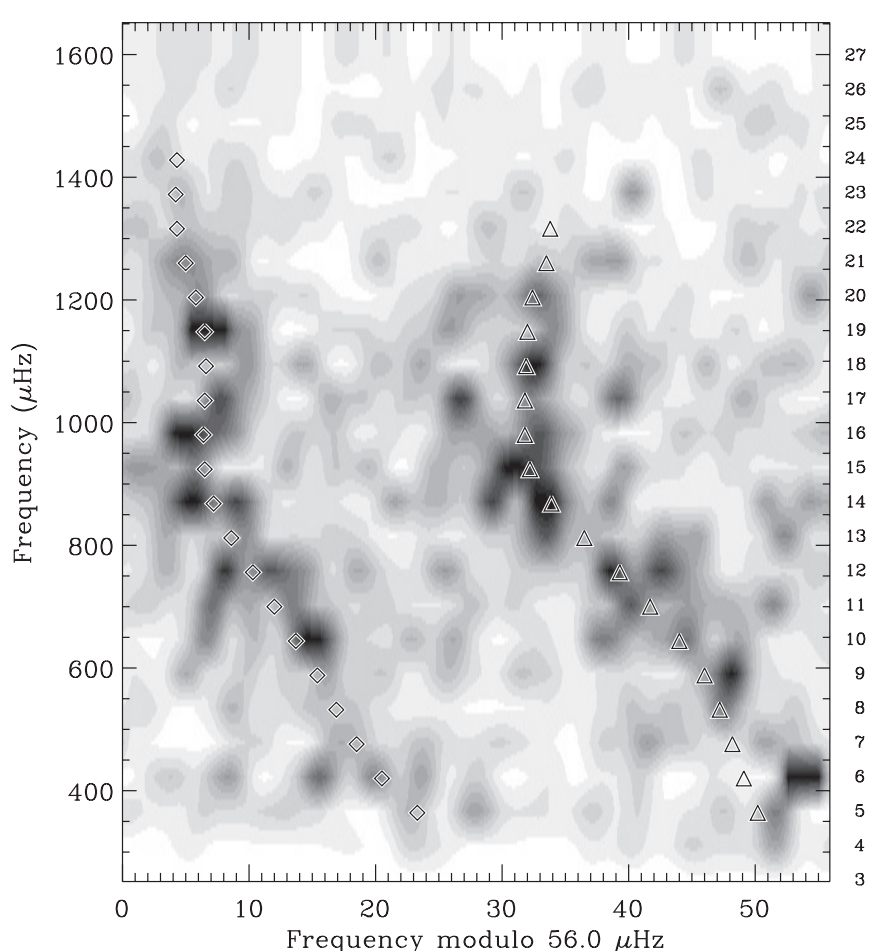

Figure 9. Centroids of the two ridges, as measured from the comb response. The grayscale shows the sidelobe-optimized power spectrum from which the peaks were calculated.
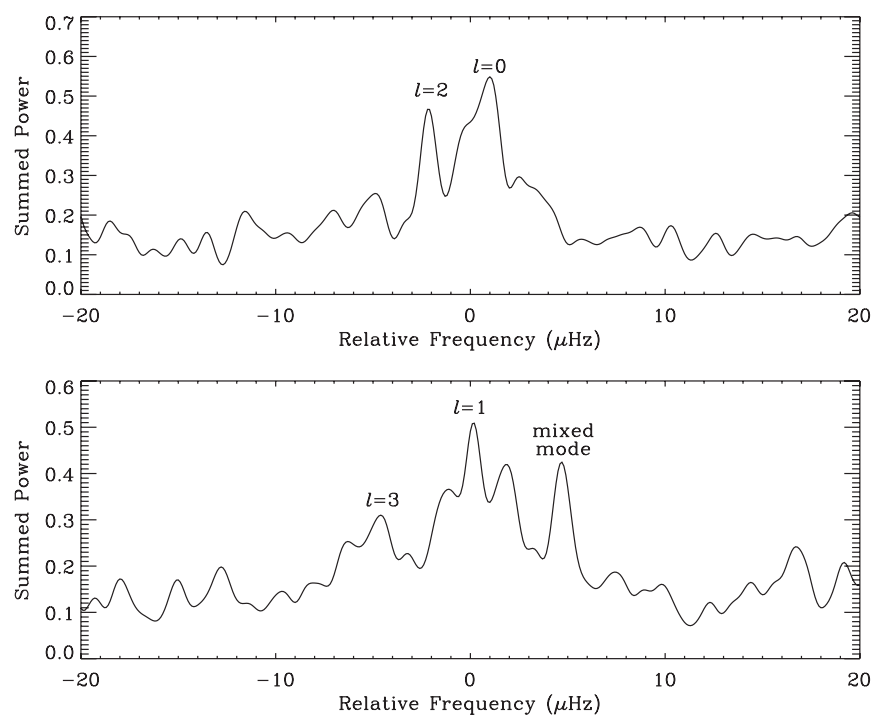

Figure 10. Power spectrum of Procyon collapsed along the ridges, over the full range of oscillations (18 orders). The upper panel shows the left-hand ridge, which we identify with modes having even degree, and the lower panel shows the right-hand ridge (odd degree). Note that the power spectrum was first smoothed slightly by convolving with a Gaussian with FWHM $0.6 \mu \mathrm{Hz}$.

In Figure 10, we show the full power spectrum of Procyon (using sidelobe-optimized weights) collapsed along the ridges. This is similar to Figure 6 except that each order was shifted before the summation, so as to align the ridge peaks (symbols in Figure 9) and hence remove the curvature. This was done separately for both the even- and odd-degree ridges, as shown in the two panels of Figure 10. The collapsed spectrum clearly shows the power corresponding to $l=0-3$, as well as the extra power from the mixed modes (for this figure, the peak at $446 \mu \mathrm{Hz}$ has not been removed).
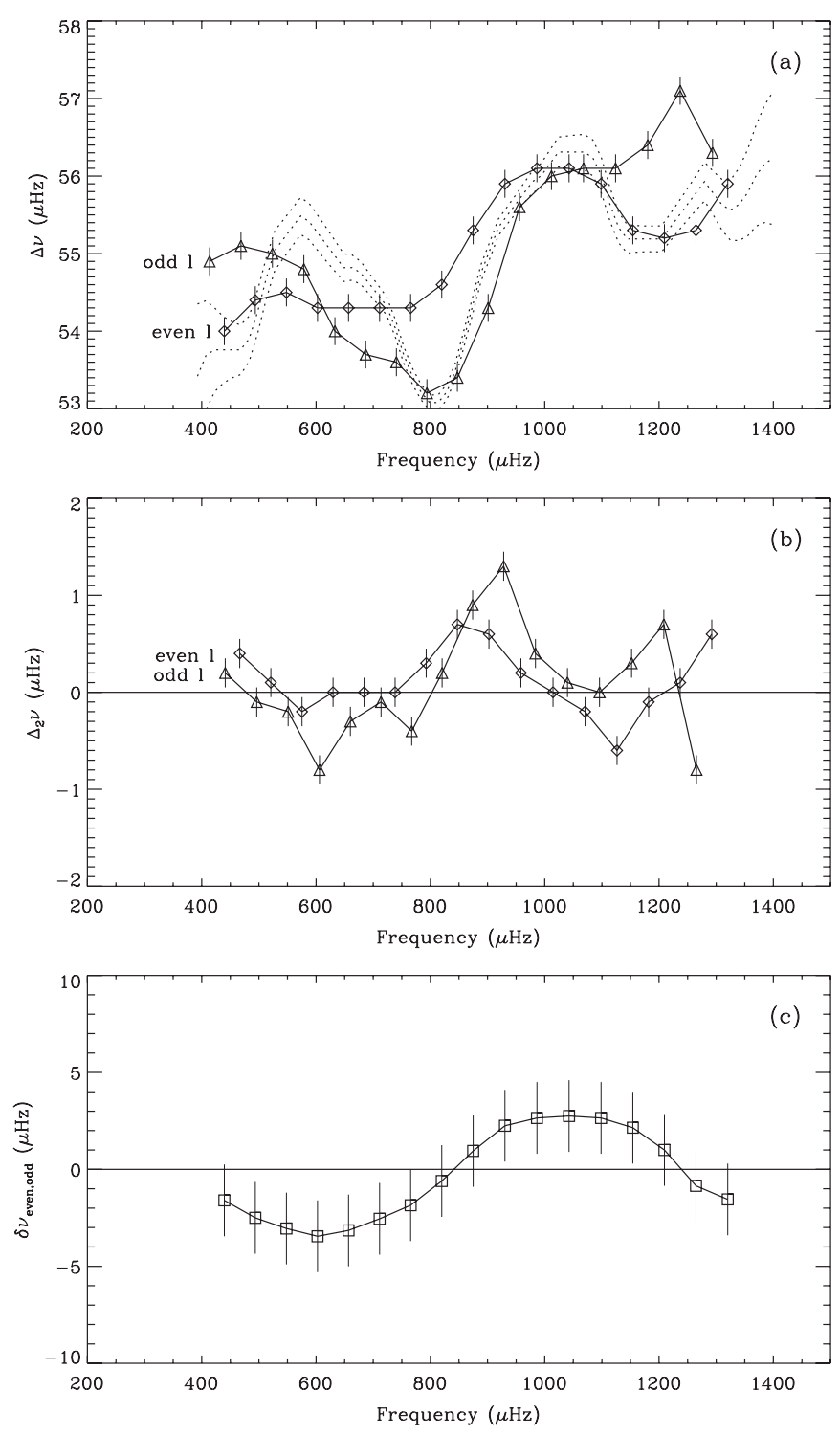

Figure 11. Symbols show the frequency separations in Procyon as a function of frequency, as measured from the ridge centroids: (a) large frequency separation, (b) second differences, and (c) small frequency separation. The dotted lines in panel $a$ show the variation in $\Delta v$ (with $\pm 1 \sigma$ range) calculated from the autocorrelation of the time series-see the text.

In Section 6 below, we use the ridges to guide our identification of the individual modes. First, however, we show that some asteroseismological inferences can be made solely from the ridges themselves. This is explained in more detail in Appendix B.

\subsection{Large Separation of the Ridges}

Figure 11(a) shows the variation with frequency of the large separation for each of the two ridges (diamonds and triangles). The smoothing across orders (Equation (6)) means that the ridge frequencies are correlated from one order to the next and so we used simulations to estimate uncertainties for the ridge centroids.

The oscillatory behavior of $\Delta v$ as a function of frequency seen in Figure 11(a) is presumably a signature of the helium ionization zone (e.g., Gough 1990). The oscillation is also seen in Figure 11(b), which shows the second differences for the two ridges, defined as follows (see Gough 1990; Ballot et al. 2004; 
Houdek \& Gough 2007):

$$
\begin{aligned}
& \Delta_{2} v_{n, \text { even }}=v_{n-1, \text { even }}-2 v_{n, \text { even }}+v_{n+1, \text { even }}, \\
& \Delta_{2} v_{n, \text { odd }}=v_{n-1, \text { odd }}-2 v_{n, \text { odd }}+v_{n+1, \text { odd }} .
\end{aligned}
$$

The period of the oscillation is $\sim 500 \mu \mathrm{Hz}$, which implies a glitch at an acoustic depth that is approximately twice the inverse of this value (Gough 1990; Houdek \& Gough 2007), namely $\sim 1000 \mathrm{~s}$. To determine this more precisely, we calculated the power spectrum of the second differences for both the odd and even ridges, and measured the highest peak. We found the period of the oscillation in the second differences to be $508 \pm 18 \mu \mathrm{Hz}$. Comparing this result with theoretical models will be the subject of a future paper.

The dotted lines in Figure 11(a) show the variation of $\Delta v$ with frequency calculated from the autocorrelation of the time series using the method of Mosser \& Appourchaux (2009; see also Roxburgh \& Vorontsov 2006). The mixed mode at $446 \mu \mathrm{Hz}$ was first removed and the smoothing filter had FWHM equal to 3 times the mean large separation. We see general agreement with the values calculated from the ridge separations. Some of the differences presumably arise because the autocorrelation analysis of the time series averages the large separation over all degrees.

\subsection{Small Separation of the Ridges}

Using only the centroids of the ridges, we can measure a small separation that is useful for asteroseismology. By analogy with $\delta v_{01}$ (see Equation (3)), we define it as the amount by which the odd ridge is offset from the midpoint of the two adjacent even ridges, with a positive value corresponding to a leftward shift (as observed in the Sun). That is,

$$
\delta v_{\text {even }, \text { odd }}=\frac{v_{n, \text { even }}+v_{n+1, \text { even }}}{2}-v_{n, \text { odd }}
$$

Figure 11(c) shows our measurements of this small separation. ${ }^{35}$ It is related in a simple way to the conventional small separations $\delta v_{01}, \delta v_{02}$, and $\delta v_{13}$ (see Appendix B for details) and so, like them, it gives information about the sound speed in the core. Our measurements of this small separation can be compared with theoretical models using the equations in Appendix B (e.g., see Christensen-Dalsgaard \& Houdek 2009).

\section{FREQUENCIES OF INDIVIDUAL MODES}

We have extracted oscillation frequencies from the time series using the standard procedure of iterative sine-wave fitting. Each step of the iteration involves finding the strongest peak in the sidelobe-optimized power spectrum and subtracting the corresponding sinusoid from the time series. Figure 12 shows the result. The two ridges are clearly visible but the finite mode lifetime causes many modes to be split into two or more peaks. We might also be tempted to propose that some of the multiple peaks are due to rotational splitting but, as shown in Appendix A, this is unlikely to be the case.

Deciding on a final list of mode frequencies with correct $l$ identifications is somewhat subjective. To guide this process, we used the ridge centroids shown in Figure 9 as well as

\footnotetext{
35 We could also define a small separation $\delta v_{\text {odd,even }}$ to be the amount by which the centroid of the even ridge is offset rightward from the midpoint of the adjacent odd ridges. This gives similar results.
}

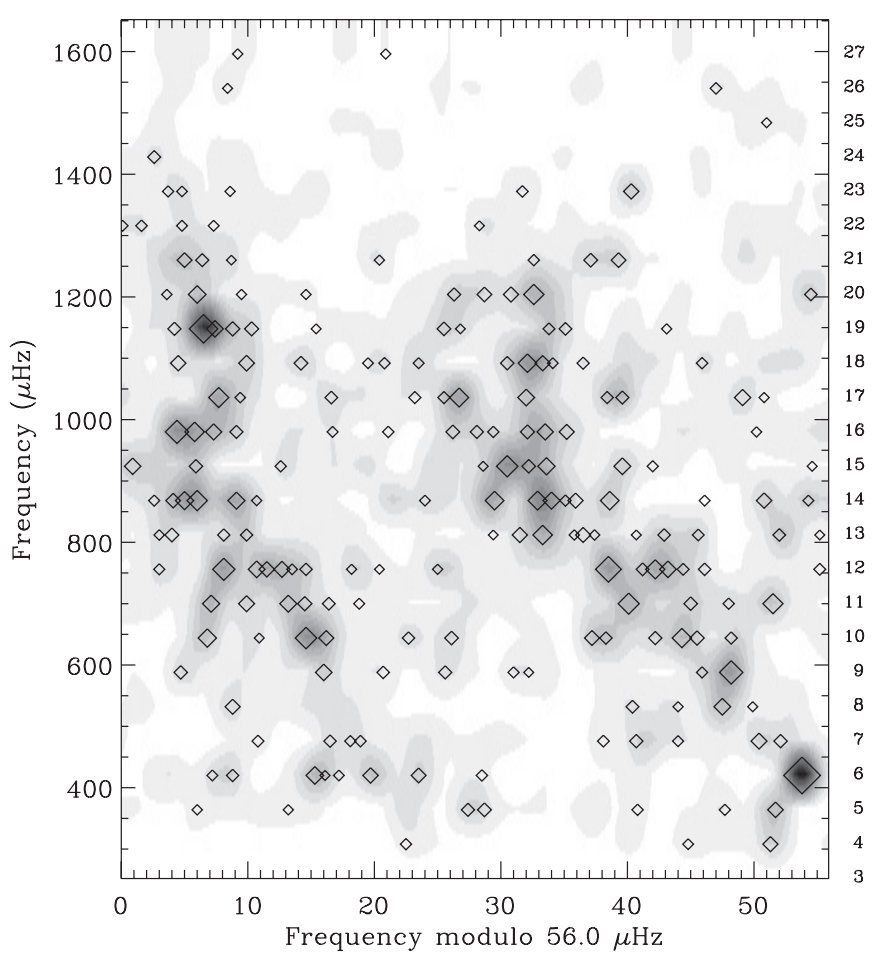

Figure 12. Peaks extracted from sidelobe-optimized power spectrum using iterative sine-wave fitting. Symbol size is proportional to amplitude (after the background noise has been subtracted). The grayscale shows the sidelobeoptimized power spectrum on which the fitting was performed, to guide the eye.

the small separations $\delta v_{02}$ and $\delta v_{13}$ from the collapsed power spectrum (see Figures 6 and 10). Each frequency extracted using iterative sine-wave fitting that lay close to a ridge was assigned an $l$ value and multiple peaks from the same mode were averaged. The final mode frequencies are listed in Table 1, while peaks with $\mathrm{S} / \mathrm{N} \geqslant 3.5$ that we have not identified are listed in Table 2. Figures 13 and 14 show these peaks overlaid on the sidelobe-optimized power spectrum. Figure 15 shows the three small separations (Equations (2)-(4)) as calculated from the frequencies listed in Table 1. The uncertainties in the mode frequencies are shown in parentheses in Table 1. These depend on the $\mathrm{S} / \mathrm{N}$ of the peak and were calibrated using simulations (e.g., see Bedding et al. 2007).

The entries in Table 2 are mostly false peaks due to noise and to residuals from the iterative sine-wave fitting, but may include some genuine modes. To check whether some of them may be daily aliases of each other or of genuine modes, we calculated the differences of all combinations of frequencies in Tables 1 and 2. The histogram of these pairwise differences was flat in the vicinity of $11.6 \mu \mathrm{Hz}$ and showed no excess, confirming that daily aliases do not contribute significantly to the list of frequencies in the tables.

We also checked whether the number peaks in Table 2 agrees with expectations. We did this by analyzing a simulated time series that matched the observations in terms of oscillations properties (frequencies, amplitudes, and mode lifetimes), noise level, window function, and distribution of weights. We extracted peaks from the simulated power spectrum using iterative sine-wave fitting, as before, and found the number of "extra" peaks (not coinciding with the oscillation ridges) to be similar to that seen in Figure 12. Finally, we remark that the peak at $408 \mu \mathrm{Hz}$ is a candidate for a mixed mode with $l=1$, given that 
Table 1

Oscillation Frequencies in Procyon (in $\mu \mathrm{Hz}$ )

\begin{tabular}{|c|c|c|c|c|}
\hline Order & $l=0$ & $l=1$ & $l=2$ & $l=3$ \\
\hline 4 & $\ldots$ & $331.3(0.8)$ & $\ldots$ & $\ldots$ \\
\hline 5 & $\ldots$ & $387.7(0.7)$ & $\ldots$ & $\ldots$ \\
\hline 6 & $415.5(0.8)$ & $445.8(0.3)$ & $411.7(0.7)$ & $\ldots$ \\
\hline 7 & $466.5(1.0)$ & $498.6(0.7)$ & $464.5(0.9)$ & $488.7(0.9)$ \\
\hline 8 & $\ldots$ & $551.5(0.7)$ & $\ldots$ & $544.4(0.9)$ \\
\hline 9 & $576.0(0.7)$ & $608.2(0.5)$ & $\ldots$ & $\ldots$ \\
\hline 10 & $630.7(0.6)$ & $660.6(0.7)$ & $627.0(1.1)$ & $653.6(0.8)$ \\
\hline 11 & $685.6(0.7)$ & $712.1(0.5)$ & $681.9(0.7)$ & $\ldots$ \\
\hline 12 & $739.2(0.7)$ & $766.5(0.5)$ & $736.2(0.5)$ & $\ldots$ \\
\hline 13 & $793.7(0.9)$ & $817.2(0.6)$ & $792.3(0.9)$ & $\ldots$ \\
\hline 14 & $849.1(0.7)$ & $873.5(0.6)$ & $845.4(0.6)$ & $869.5(0.6)$ \\
\hline 15 & $901.9(0.8)$ & $929.2(0.7)$ & $\ldots$ & $926.6(0.6)$ \\
\hline 16 & $957.8(0.6)$ & $985.3(0.7)$ & $956.4(0.5)$ & $980.4(0.9)$ \\
\hline 17 & $1015.8(0.6)$ & $1040.0(0.7)$ & $\ldots$ & $1034.5(0.7)$ \\
\hline 18 & $1073.9(0.7)$ & $1096.5(0.7)$ & $1068.5(0.7)$ & $\ldots$ \\
\hline 19 & $1126.7(0.5)$ & $1154.6(0.9)$ & $1124.3(0.9)$ & $\ldots$ \\
\hline 20 & $1182.0(0.7)$ & $1208.5(0.6)$ & $1179.9(1.0)$ & $\ldots$ \\
\hline 21 & $1238.3(0.9)$ & $1264.6(1.0)$ & $1237.0(0.8)$ & $\ldots$ \\
\hline 22 & $1295.2(1.0)$ & $\ldots$ & $1292.8(1.0)$ & $\ldots$ \\
\hline 23 & $1352.6(1.1)$ & $1375.7(1.0)$ & $1348.2(1.0)$ & $\ldots$ \\
\hline
\end{tabular}

it lies in the same order as the previously identified mixed mode at $446 \mu \mathrm{Hz}$ (note that we expect one extra $l=1$ mode to occur at an avoided crossing).

The modes listed in Table 1 span 20 radial orders and more than a factor of 4 in frequency. This range is similar to that obtained from long-term studies of the Sun (e.g., Broomhall et al. 2009) and is unprecedented in asteroseismology. It was made possible by the unusually broad range of excited modes in Procyon and the high $\mathrm{S} / \mathrm{N}$ of our data. Since the stellar background at low frequencies in intensity measurements is expected to be much higher than for velocity measurements, it seems unlikely that even the best data from the Kepler Mission will return such a wide range of frequencies in a single target.

\section{MODE LIFETIMES}

As discussed in Section 2, if the time series is sufficiently long then damping causes each mode in the power spectrum to be split into a series of peaks under a Lorentzian envelope having FWHM $\Gamma=1 /(\pi \tau)$, where $\tau$ is the mode lifetime. Our observations of Procyon are not long enough to resolve the modes into clear Lorentzians, and instead we see each mode as a small number of peaks (sometimes one). Furthermore, the centroid of these peaks may be offset from the position of the true mode, as illustrated in Figure 1 of Anderson et al. (1990). This last feature allows one to use the scatter of the extracted frequencies about smooth ridges in the échelle diagram, calibrated using simulations, to estimate the mode lifetime (Kjeldsen et al. 2005; Bedding et al. 2007). That method cannot be applied to Procyon because the $l=0$ and $l=2$ ridges are not well resolved and the $l=1$ ridge is affected by mixed modes.

Rather than looking at frequency shifts, we have estimated the mode lifetime from the variations in mode amplitudes (again calibrated using simulations). This method is less precise but has the advantage of being independent of the mode identifications (e.g., Leccia et al. 2007; Carrier et al. 2007; Bedding et al. 2007). In Paper I, we calculated the smoothed amplitude curve for Procyon in 10 two-day segments and used the fluctuations about the mean to make a rough estimate of the mode lifetime:
Table 2

Unidentified Peaks with $\mathrm{S} / \mathrm{N} \geqslant 3.5$

\begin{tabular}{cc}
\hline $\begin{array}{c}v \\
(\mu \mathrm{Hz})\end{array}$ & $\mathrm{S} / \mathrm{N}$ \\
\hline $407.6(0.8)$ & 3.5 \\
$512.8(0.8)$ & 3.6 \\
$622.8(0.6)$ & 4.3 \\
$679.1(0.7)$ & 4.0 \\
$723.5(0.6)$ & 4.7 \\
$770.5(0.7)$ & 4.1 \\
$878.5(0.6)$ & 4.4 \\
$890.8(0.7)$ & 3.6 \\
$935.6(0.7)$ & 3.9 \\
$1057.2(0.7)$ & 3.7 \\
$1384.3(0.7)$ & 3.6 \\
\hline
\end{tabular}

$\tau=1.5_{-0.8}^{+1.9}$ days. We have attempted to improve on that estimate by considering the amplitude fluctuations of individual modes, as has been done for the Sun (e.g., Toutain \& Fröhlich 1992; Baudin et al. 1996; Chang \& Gough 1998), but were not able to produce well-calibrated results for Procyon.

Instead, we have measured the "peakiness" of the power spectrum (see Bedding et al. 2007) by calculating the ratio between the square of the mean amplitude of the 15 highest peaks in the range 500-1300 $\mu \mathrm{Hz}$ (found by iterative sine-wave fitting) and the mean power in the same frequency range. The value for this ratio from our observations of Procyon is 6.9. We made a large number of simulations (3600) having a range of mode lifetimes and with the observed frequency spectrum, noise level, window function, and weights. Comparing the simulations with the observations led to a mode lifetime for Procyon of $1.29_{-0.49}^{+0.55}$ days.

This agrees with the value found in Paper I but is more precise, confirming that modes in Procyon are significantly more shortlived than those of the Sun. As discussed in Section 2, the dominant modes in the Sun have lifetimes of 2-4 days (e.g., Chaplin et al. 1997). The tendency for hotter stars to have shorter mode lifetimes has recently been discussed by Chaplin et al. (2009).

\section{FITTING TO THE POWER SPECTRUM}

Extracting mode parameters by fitting directly to the power spectrum is widely used in helioseismology, where the time series extends continuously for months or even years, and so the individual modes are well resolved (e.g., Anderson et al. 1990). Mode fitting has not been applied to ground-based observations of solar-type oscillations because these data typically have shorter durations and significant gaps. Global fitting has been carried out on spacecraft data, beginning with the 50-day time series of $\alpha$ Cen A taken with the WIRE spacecraft (Fletcher et al. 2006) and the 60-day light curve of HD 49933 from CoRoT (Appourchaux et al. 2008). Our observations of Procyon are much shorter than either of these cases but, given the quality of the data and the spectral window, we considered it worthwhile to attempt a fit.

Global fits to the Procyon power spectrum were made by several of us. Here, we present results from a fit using a Bayesian approach (e.g., Gregory 2005), which allowed us to include in a straightforward way our prior knowledge of the oscillation properties. The parameters to be extracted were the frequencies, heights, and linewidths of the modes. To obtain the marginal probability distributions of these parameters 

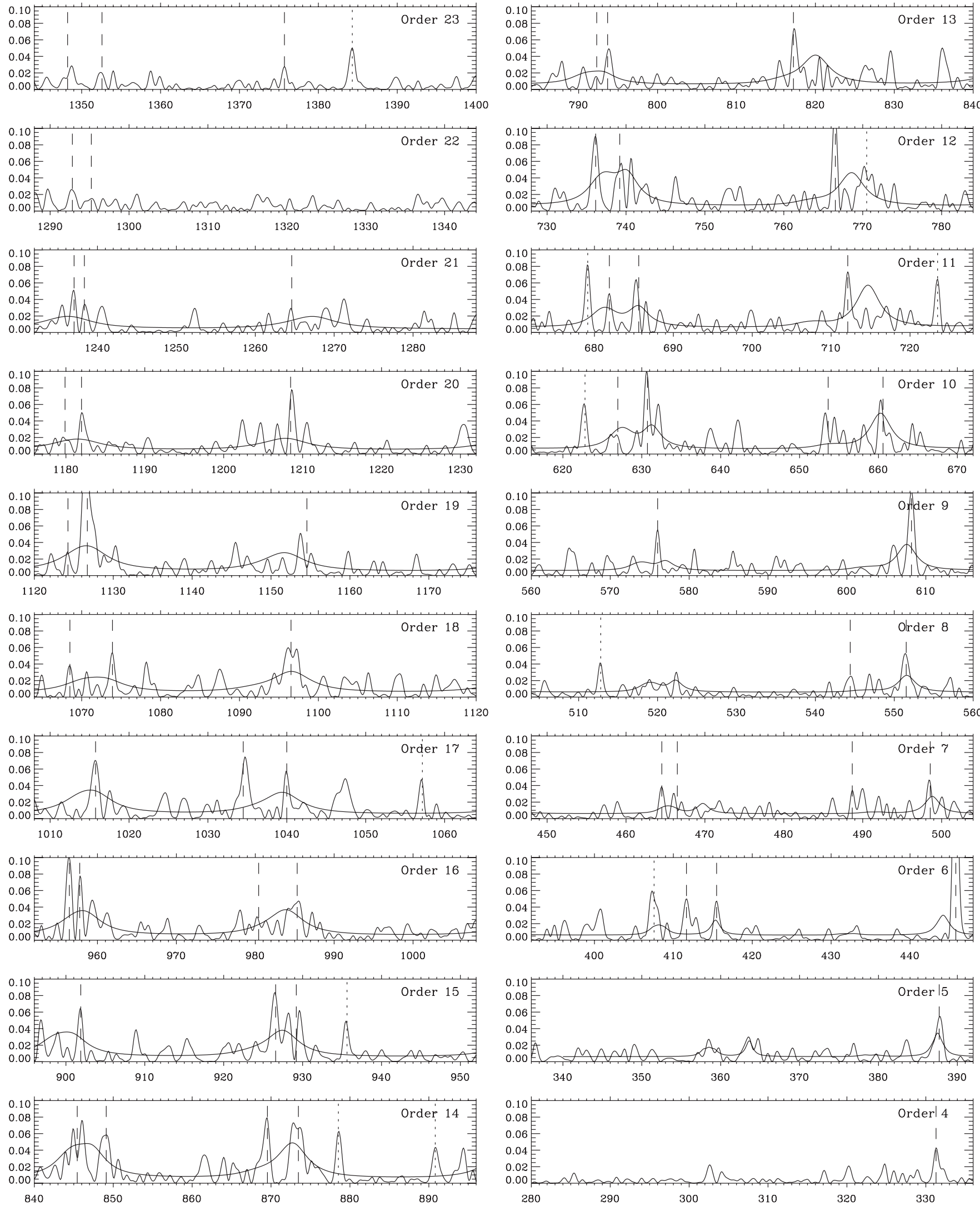

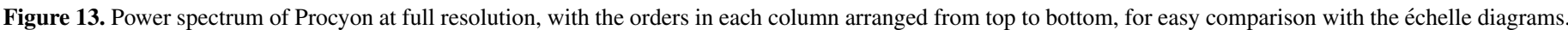

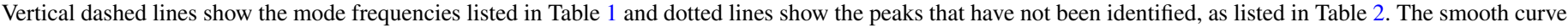
shows the global fit to the power spectrum for Scenario B (see Section 8).

and their associated uncertainties, we employed an Automated Parallel Tempering Markov Chain Monte Carlo (APT MCMC) algorithm. It implements the Metropolis-Hastings sampler by performing a random walk in parameter space while drawing 


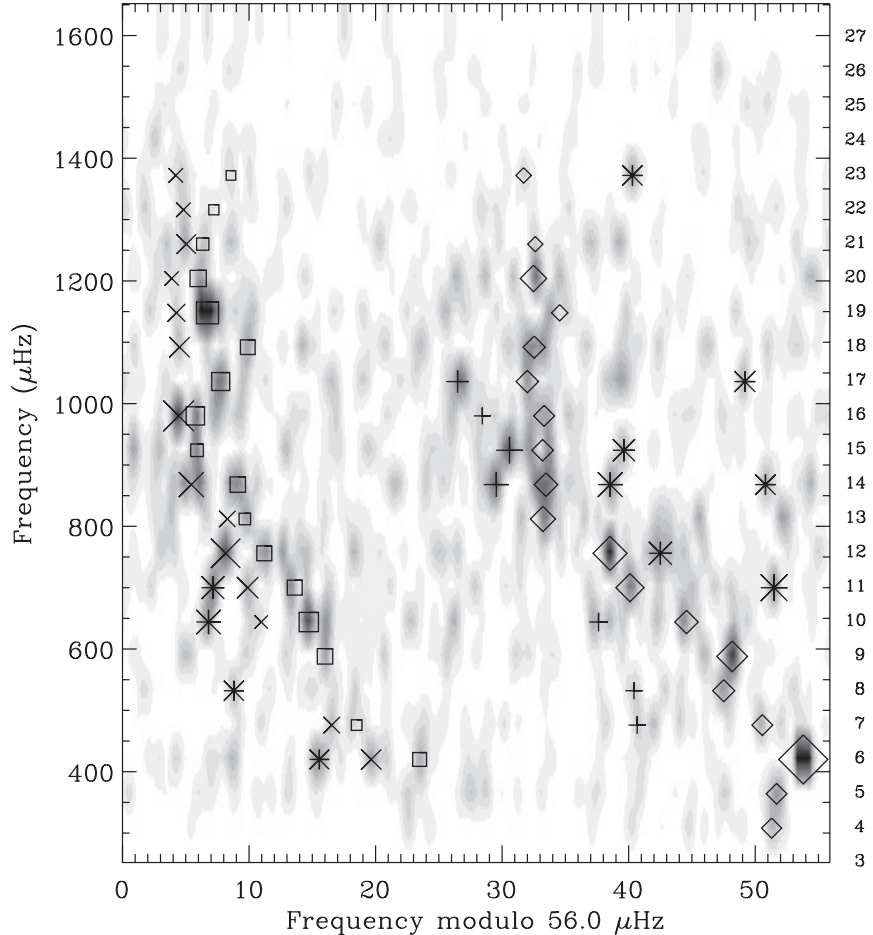

Figure 14. Power spectrum of Procyon overlaid with mode frequencies listed in Table 1. Symbols indicate angular degree (squares: $l=0$; diamonds: $l=1$; crosses: $l=2$; pluses: $l=3$ ). Asterisks show the peaks that have not been identified, as listed in Table 2.

samples from the posterior distribution (Gregory 2005). Further details of our implementation of the algorithm will be given elsewhere (T. L. Campante et al. 2010, in preparation).

The details of the fitting are as follows.

1. The fitting was performed over 17 orders (5-21) using the sidelobe-optimized power spectrum. In each order, we fitted modes with $l=0,1$, and 2 , with each individual profile being described by a symmetric Lorentzian with FWHM $\Gamma$ and height $H$. The mode frequencies were constrained to lie close to the ridges and to have only small jumps from one order to the next (a Gaussian prior with $\sigma=3 \mu \mathrm{Hz}$ ). The $\mathrm{S} /$ Ns of modes with $l=3$ were too low to permit a fit. In order to take their power into account, we included them in the model with their frequencies fixed by the asymptotic relation (Equation (1)).

2. The data are not good enough to provide a useful estimate of the linewidth of every mode, or even of every order. Therefore, the linewidth was parameterized as a linear function of frequency, defined by two parameters $\Gamma_{600}$ and $\Gamma_{1200}$, which are the values at 600 and $1200 \mu \mathrm{Hz}$. These parameters were determined by the fit, in which both were assigned a uniform prior in the range $0-10 \mu \mathrm{Hz}$.

3 . The height of each mode is related to the linewidth and amplitude according to (Chaplin et al. 2005):

$$
H=\frac{2 A^{2}}{\pi \Gamma} .
$$

The amplitudes $A$ of the modes were determined as follows. For the radial modes $(l=0)$, we used the smoothed amplitude curve measured from our observations, as shown in Figure 10 of Paper I. The amplitudes of the non-radial modes $(l=1-3)$ were then calculated from the radial

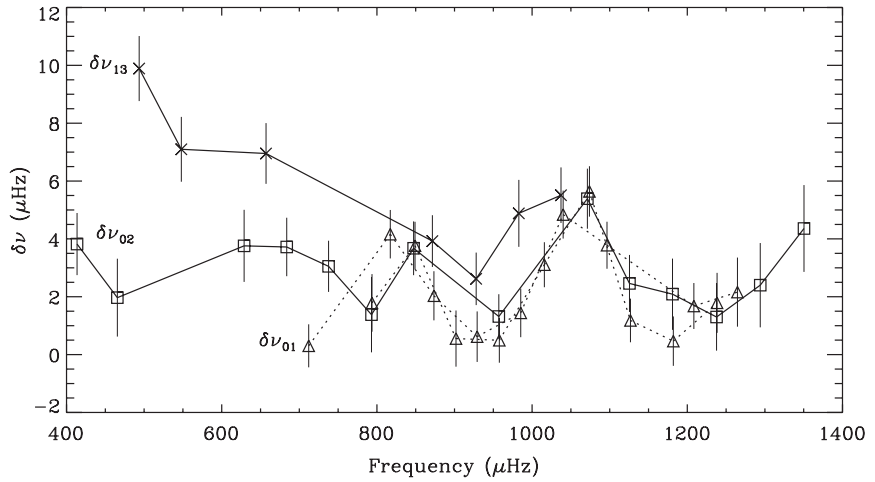

Figure 15. Small frequency separations in Procyon, as measured from the mode frequencies listed in Table 1.

Table 3

Frequencies from Global Fit Using Scenario B (in $\mu \mathrm{Hz}$, with $-/+$ Uncertainties)

\begin{tabular}{cccc}
\hline \hline Order & $l=0$ & $l=1$ & $l=2$ \\
\hline 5 & $363.6(0.8 / 0.9)$ & $387.5(0.6 / 0.6)$ & $358.5(1.3 / 1.2)$ \\
6 & $415.3(3.3 / 1.0)$ & $\ldots$ & $408.1(1.0 / 3.7)$ \\
7 & $469.7(1.6 / 2.1)$ & $498.8(0.7 / 0.8)$ & $465.3(1.1 / 1.3)$ \\
8 & $522.3(1.4 / 1.4)$ & $551.6(0.8 / 0.7)$ & $519.0(1.5 / 1.6)$ \\
9 & $577.0(1.6 / 2.5)$ & $607.6(0.6 / 0.7)$ & $573.9(2.2 / 2.8)$ \\
10 & $631.3(0.8 / 0.8)$ & $660.3(1.0 / 1.3)$ & $627.4(2.1 / 2.8)$ \\
11 & $685.6(1.2 / 1.6)$ & $714.7(1.4 / 1.2)$ & $681.2(2.3 / 1.9)$ \\
12 & $740.1(1.6 / 1.7)$ & $768.6(0.9 / 1.0)$ & $737.0(1.5 / 1.7)$ \\
13 & $793.2(1.3 / 1.7)$ & $820.0(1.7 / 1.2)$ & $790.9(2.0 / 1.9)$ \\
14 & $847.3(1.2 / 1.4)$ & $872.7(1.1 / 0.9)$ & $844.7(1.7 / 1.5)$ \\
15 & $901.0(1.8 / 1.7)$ & $927.5(0.8 / 0.8)$ & $898.6(2.1 / 2.1)$ \\
16 & $958.7(1.4 / 1.1)$ & $983.9(1.0 / 1.3)$ & $957.2(1.0 / 1.3)$ \\
17 & $1015.9(1.5 / 1.8)$ & $1039.5(1.6 / 1.7)$ & $1014.0(1.8 / 2.4)$ \\
18 & $1073.2(1.5 / 2.2)$ & $1096.6(1.1 / 1.0)$ & $1070.3(2.2 / 2.3)$ \\
19 & $1127.2(1.0 / 1.3)$ & $1151.8(1.4 / 1.4)$ & $1125.9(1.3 / 1.4)$ \\
20 & $1182.3(1.5 / 1.4)$ & $1207.9(1.4 / 1.1)$ & $1180.5(1.6 / 1.6)$ \\
21 & $1236.9(1.7 / 1.6)$ & $1267.4(1.7 / 1.5)$ & $1235.5(2.0 / 1.7)$ \\
\hline
\end{tabular}

modes using the ratios given in Table 1 of Kjeldsen et al. (2008a), namely, $S_{0}: S_{1}: S_{2}: S_{3}=1.00: 1.35: 1.02$ : 0.47 .

4. The background was fitted as a flat function.

5. We calculated the rotationally split profiles of the nonradial modes using the description given by Gizon \& Solanki (2003). The inclination angle of the rotation axis was fixed at $31^{\circ}$, which is the inclination of the binary orbit (Girard et al. 2000) and, as discussed in Paper I (Section 4.1), is consistent with the rotational modulation of the velocity curve. The rotational splitting was fixed at $0.7 \mu \mathrm{Hz}$, which was chosen to match the observed value of $v \sin i=3.16 \mathrm{~km} \mathrm{~s}^{-1}$ (Allende Prieto et al. 2002), given the known radius of the star. As discussed in Appendix A, choosing different values for the inclination (and hence the splitting) does not affect the mode profile, assuming reasonable values of the linewidth.

We carried out the global fit using both scenarios discussed in Section 4. The fit for Scenario B is shown as the smooth curve in Figure 13 and the fitted frequencies are given in Table 3. Note that the mixed mode at $446 \mu \mathrm{Hz}$ was not properly fitted because it lies too far from the ridge (see point 1 above). To check the agreement with the results discussed in Section 6, we examined the differences betweens the frequencies in Tables 1 and 3. We found a reduced $\chi^{2}$ of 0.74 , which indicates good agreement. A 


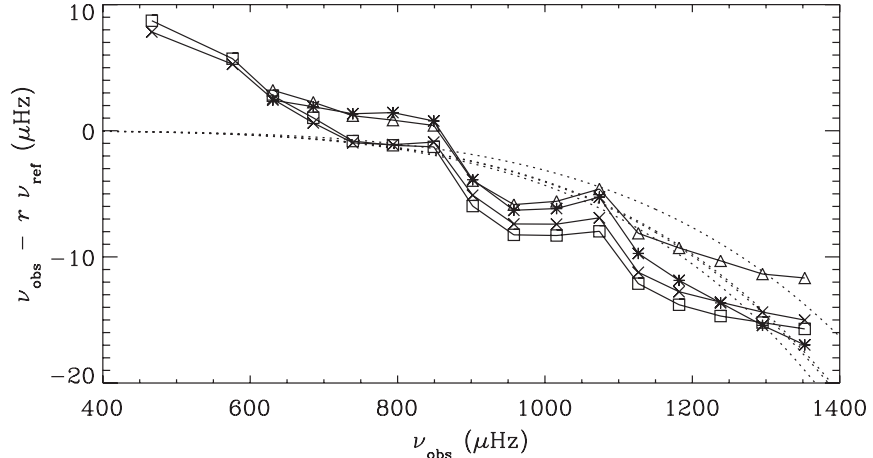

Figure 16. Difference between observed frequencies of radial modes in Procyon and those of scaled models. The symbols indicate different models, as follows: squares from Chaboyer et al. (1999, Table 2), crosses from Di Mauro \& Christensen-Dalsgaard (2001), asterisks from Kervella et al. (2004, Table 4), and triangles from Eggenberger et al. (2005, model M1a). In each case, the dotted curve shows the correction calculated using Equation (4) of Kjeldsen et al. (2008b)

value less than 1 is not surprising given that both methods were constrained to find modes close to the ridges.

The fitted linewidths (assumed to be a linear function of frequency, as described above) gave mode lifetimes of $1.5 \pm$ 0.4 days at $600 \mu \mathrm{Hz}$ and $0.6 \pm 0.3$ days at $1200 \mu \mathrm{Hz}$. These agree with the single value of $1.29_{-0.49}^{+0.55}$ days found above (Section 7), and indicate that the lifetime increases toward lower frequencies, as is the case for the Sun and for the Ftype CoRoT targets HD 49933 (Benomar et al. 2009b) and HD 181420 (Barban et al. 2009).

We also carried out the global fit using Scenario A. We found through Bayesian model selection that Scenario A was statistically favored over Scenario B by a factor of 10:1. This factor classifies as "significant" on the scale of Jeffreys (1961; see Table 1 of Liddle 2009). On the same scale, posterior odds of at least $\sim 13: 1$ are required for a classification of "strong to very strong," and "decisive" requires at least $\sim 150: 1$. In our Bayesian fit to Procyon, the odds ratio in favor of Scenario A did not exceed 13:1, even when different sets of priors were imposed.

In light of the strong arguments given in Section 4 in favor of Scenario B, we do not consider the result from Bayesian model selection to be sufficiently compelling to cause us to reverse our identification. Of course, it is possible that Scenario A is correct and, for completeness, we show these fitted frequencies in Table 4. The fit using Scenario A gave mode lifetimes of $0.9 \pm 0.2$ days at $600 \mu \mathrm{Hz}$ and $1.0 \pm 0.3$ days at $1200 \mu \mathrm{Hz}$.

\section{PRELIMINARY COMPARISON WITH MODELS}

A detailed comparison of the observed frequencies of Procyon with theoretical models is beyond the scope of this paper, but we will make some preliminary comments on the systematic offset between the two. It is well established that incorrect modeling of the surface layers of the Sun is responsible for discrepancies between the observed and calculated oscillation frequencies (Christensen-Dalsgaard et al. 1988; Dziembowski et al. 1988; Rosenthal et al. 1999; Li et al. 2002).

To address this problem for other stars, Kjeldsen et al. (2008b) proposed an empirical correction to be applied to model frequencies that takes advantage of the fact that the offset between observations and models is independent of $l$ and goes to zero with decreasing frequency. They measured the offset for the Sun to be a power law with exponent $b=4.9$ and applied

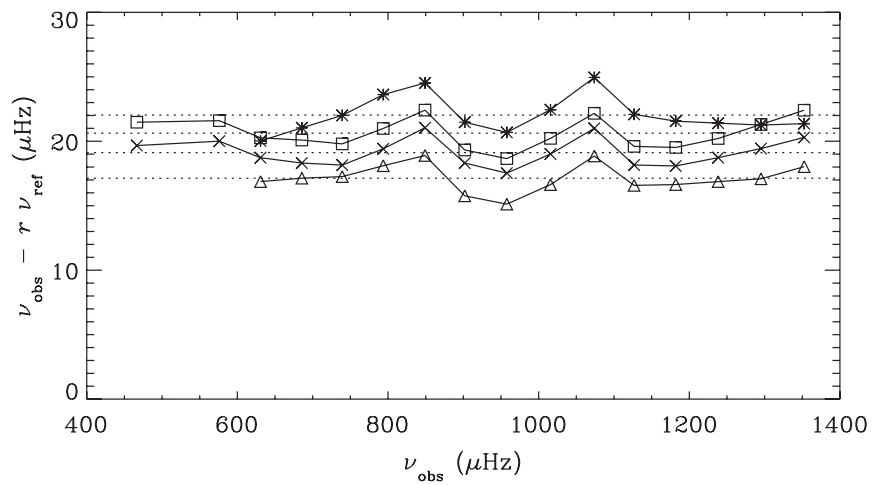

Figure 17. Same as Figure 16, but with a constant near-surface correction $(b=0)$.

Table 4

Frequencies from Global Fit Using Scenario A (in $\mu \mathrm{Hz}$, with $-/+$ Uncertainties)

\begin{tabular}{lccr}
\hline \hline Order & $l=0$ & $l=1$ & $l=2$ \\
\hline 5 & $387.7(1.9 / 1.8)$ & $361.9(1.8 / 2.0)$ & $385.1(1.9 / 2.6)$ \\
6 & $\ldots$ & $412.5(1.7 / 2.3)$ & $439.3(2.6 / 2.6)$ \\
7 & $498.7(1.1 / 1.6)$ & $467.6(1.4 / 1.3)$ & $493.2(2.6 / 2.0)$ \\
8 & $552.2(1.5 / 1.5)$ & $520.7(1.2 / 1.3)$ & $549.3(2.2 / 2.0)$ \\
9 & $607.8(1.0 / 0.9)$ & $576.2(1.1 / 1.4)$ & $605.4(2.2 / 2.3)$ \\
10 & $661.3(1.3 / 1.5)$ & $631.1(0.7 / 0.8)$ & $657.1(1.7 / 1.6)$ \\
11 & $716.8(1.3 / 1.7)$ & $684.7(1.2 / 1.2)$ & $712.6(1.2 / 1.2)$ \\
12 & $769.9(1.2 / 1.3)$ & $739.1(1.1 / 1.2)$ & $766.6(1.4 / 1.4)$ \\
13 & $822.7(1.9 / 2.7)$ & $792.9(1.3 / 1.3)$ & $817.8(1.3 / 1.4)$ \\
14 & $874.5(1.3 / 1.3)$ & $846.4(0.9 / 0.8)$ & $869.9(1.6 / 1.3)$ \\
15 & $928.8(1.2 / 1.2)$ & $900.0(1.3 / 1.4)$ & $925.9(1.3 / 1.1)$ \\
16 & $985.1(1.0 / 1.1)$ & $958.2(0.8 / 0.8)$ & $980.9(1.9 / 1.6)$ \\
17 & $1043.4(2.8 / 2.8)$ & $1015.7(1.0 / 0.9)$ & $1035.2(1.0 / 0.8)$ \\
18 & $1097.6(1.5 / 0.9)$ & $1072.5(1.1 / 1.2)$ & $1091.8(3.7 / 4.2)$ \\
19 & $1153.7(0.9 / 0.8)$ & $1126.9(0.5 / 0.6)$ & $1146.8(1.3 / 1.0)$ \\
20 & $1209.1(0.8 / 0.9)$ & $1181.8(1.0 / 0.9)$ & $1204.8(1.3 / 1.4)$ \\
21 & $1269.2(1.0 / 1.1)$ & $1237.1(0.9 / 0.9)$ & $1264.8(1.5 / 1.5)$ \\
\hline
\end{tabular}

this correction to the radial modes of other stars, finding very good results that allowed them to estimate mean stellar densities very accurately (better than $0.5 \%$ ).

We have applied this method to Procyon, comparing our observed frequencies for the radial modes with various published models to determine the scaling factor $r$ and the offset (see Kjeldsen et al. 2008b for details of the method). The results are shown in Figure 16. Interestingly, the offset between the observations and scaled models does not go to zero with decreasing frequency. This contrasts with the G- and K-type stars investigated by Kjeldsen et al. (2008b), namely, the Sun, $\alpha$ Cen A and $\mathrm{B}$, and $\beta$ Hyi.

The method of Kjeldsen et al. (2008b) assumes the correction to be applied to the models to have the same form as in the Sun, namely, a power law with an exponent of $b=4.9$. The fit in Figure 16 is poor and is not improved by modest adjustments to $b$. Instead, the results seem to imply an offset that is constant. Setting $b=0$ and repeating the calculations produce the results shown in Figure 17, where we indeed see a roughly constant offset between the models and the observations of about $20 \mu \mathrm{Hz}$.

As a check, we can consider the density implied for Procyon. The stellar radius can be calculated from the interferometric radius and the parallax. The angular diameter of $5.404 \pm$ 0.031 mas (Aufdenberg et al. 2005, their Table 7) and the revised Hipparcos parallax of $285.93 \pm 0.88$ mas (van Leeuwen 2007) gives a radius of $2.041 \pm 0.015 R_{\odot}$. 
Procyon is in a binary system (the secondary is a white dwarf), allowing the mass to be determined from astrometry. Girard et al. (2000) found a value of $1.497 \pm 0.037 M_{\odot}$, while Gatewood \& Han (2006) found $1.431 \pm 0.034 M_{\odot}$ (see Guenther et al. 2008 for further discussion).

The density obtained using the fits shown in Figure 16 is in the range $0.255-0.258 \mathrm{~g} \mathrm{~cm}^{-3}$. Combining with the radius implies a mass in the range 1.54-1.56 $M_{\odot}$. The density obtained using the fits shown in Figure 17 is in the range $0.242-0.244 \mathrm{~g} \mathrm{~cm}^{-3}$, implying a mass of 1.46-1.48 $M_{\odot}$. The latter case seems to be in much better agreement with the astrometrically determined mass, lending some support to the idea that the offset is constant.

We can also consider the possibility that our mode identification is wrong and that Scenario A is the correct one (see Sections 4 and 8). With this reversed identification, the radial modes in Procyon are those in Table 1 listed as having $l=1$. Assuming these to be radial modes, the offset between them and the model frequencies is again constant, as we would expect, but this time with a mean value close to zero. The implied density for Procyon is again consistent with the observed mass and radius.

The preceding discussion makes it clear that the correction that needs to be applied to models of Procyon is very different from that for the Sun and other cool stars, regardless of whether Scenario B or A is correct. In particular, the substantial nearly constant offset implied by Figure 16 would indicate errors in the modeling extending well beyond the near-surface layers. We also note that in terms of the asymptotic expression (Equation (1)) a constant offset would imply an error in the calculation of $\epsilon$.

\section{CONCLUSION}

We have analyzed results from a multi-site campaign on Procyon that obtained high-precision velocity observations over more than three weeks (Paper I). We developed a new method for adjusting the weights in the time series that allowed us to minimize the sidelobes in the power spectrum that arise from diurnal gaps and so to construct an échelle diagram that shows two clear ridges of power. To identify the odd and even ridges, we summed the power across several orders. We found structures characteristic of $l=0$ and 2 in one ridge and $l=1$ and 3 in the other. This identification was confirmed by comparing our Procyon data in a scaled échelle diagram (Bedding \& Kjeldsen 2010) with other stars for which the ridge identification is known. We showed that the frequencies of the ridge centroids and their large and small separations are easily measured and are useful diagnostics for asteroseismology. In particular, an oscillation in the large separation appears to indicate a glitch in the sound-speed profile at an acoustic depth of $\sim 1000 \mathrm{~s}$.

We identify a strong narrow peak at $446 \mu \mathrm{Hz}$, which falls slightly away from the $l=1$ ridge, as a mixed mode. In Table 1, we give frequencies, extracted using iterative sinewave fitting, for 55 modes with angular degrees $l$ of $0-3$. These cover 20 radial orders and a factor of more than 4 in frequency, which reflects the broad range of excited modes in Procyon and the high $\mathrm{S} / \mathrm{N}$ of our data, especially at low frequencies. Intensity measurements will suffer from a much higher stellar background at low frequencies, making it unlikely that even the best data from the Kepler Mission will yield the wide range of frequencies found here. This is a strong argument in favor of continuing efforts toward ground-based Doppler studies, such as the Stellar Observations Network Group (SONG; Grundahl et al. 2008), which is currently under construction, and the proposed Antarctic instrument Seismic Interferometer to Measure Oscillations in the Interior of Stars (SIAMOIS; Mosser et al. 2008).

We estimated the mean lifetime of the modes by comparing the "peakiness" of the power spectrum with simulations and found a value of $1.29_{-0.49}^{+0.55}$ days, significantly below that of the Sun. A global fit to the power spectrum using Bayesian methods confirmed this result and provided evidence that the lifetime increases toward lower frequencies. It also casts some doubt on the mode identifications. We still favor the identification discussed above, but leave open the possibility that this may need to be reversed. Finally, comparing the observed frequencies of radial modes in Procyon with published theoretical models showed an offset that appears to be constant with frequency, making it very different from that seen in the Sun and other cool stars. Detailed comparisons of our results with theoretical models will be carried out in future papers.

We would be happy to make the data presented in this paper available on request.

This work was supported financially by the Australian Research Council, the Danish Natural Science Research Council, the Swiss National Science Foundation, NSF grant AST9988087 (R.P.B.), and by SUN Microsystems. We gratefully acknowledge support from the European Helio- and Asteroseismology Network (HELAS), a major international collaboration funded by the European Commission's Sixth Framework Programme.

\section{APPENDIX A}

\section{ROTATIONAL SPLITTING}

We expect non-radial modes to be split due to the rotation of the star. The rotation period of Procyon is not known, although slow variations in our velocity observations (Paper I) indicated a value of either 10.3 days or twice that value. The projected rotational velocity has been measured spectroscopically. Allende Prieto et al. (2002) determined a value of $v \sin i=3.16 \pm 0.50 \mathrm{~km} \mathrm{~s}^{-1}$, although they note that the actual value may be lower by about $0.5 \mathrm{~km} \mathrm{~s}^{-1}$.

Gizon \& Solanki (2003) have studied the effect of rotation on the profiles of solar-like oscillations as a function of inclination and mode lifetime (see also Ballot et al. 2006). We have repeated their calculations for our observations of Procyon (with sidelobe-optimized weights). The results are shown in Figure 18, which shows the effects of rotational splitting, inclination angle, and mode lifetime on the theoretical profile of the modes. ${ }^{36}$ Note that the calculations do not include the stochastic nature of the excitation and so the function shown here should properly be called the expectation value of the power spectrum, also known as the limit spectrum. Figure 18 is similar to Figure 2 of Gizon \& Solanki (2003) except that instead of fixing the rotation period, we have fixed $v \sin i$ to be the measured value. For $l=0$, the profile does not depend on the inclination angle, while for $l=1$, 2 , and 3 the solid and dashed lines show calculations for $i=30^{\circ}$ ( $P_{\mathrm{rot}}=16.4$ days) and $i=80^{\circ}$ ( $P_{\mathrm{rot}}=32.3$ days), respectively. In each panel, results are shown for three values of the mode lifetime: 1.5 days (top curve), 3 days (middle curve), and infinite (bottom curve). For each mode lifetime, the curves for different $i$ and $l$ are all normalized to have the same area.

\footnotetext{
${ }^{36}$ Note that we have made the quite reasonable assumption that the internal rotation has a similar period to the surface rotation.
} 

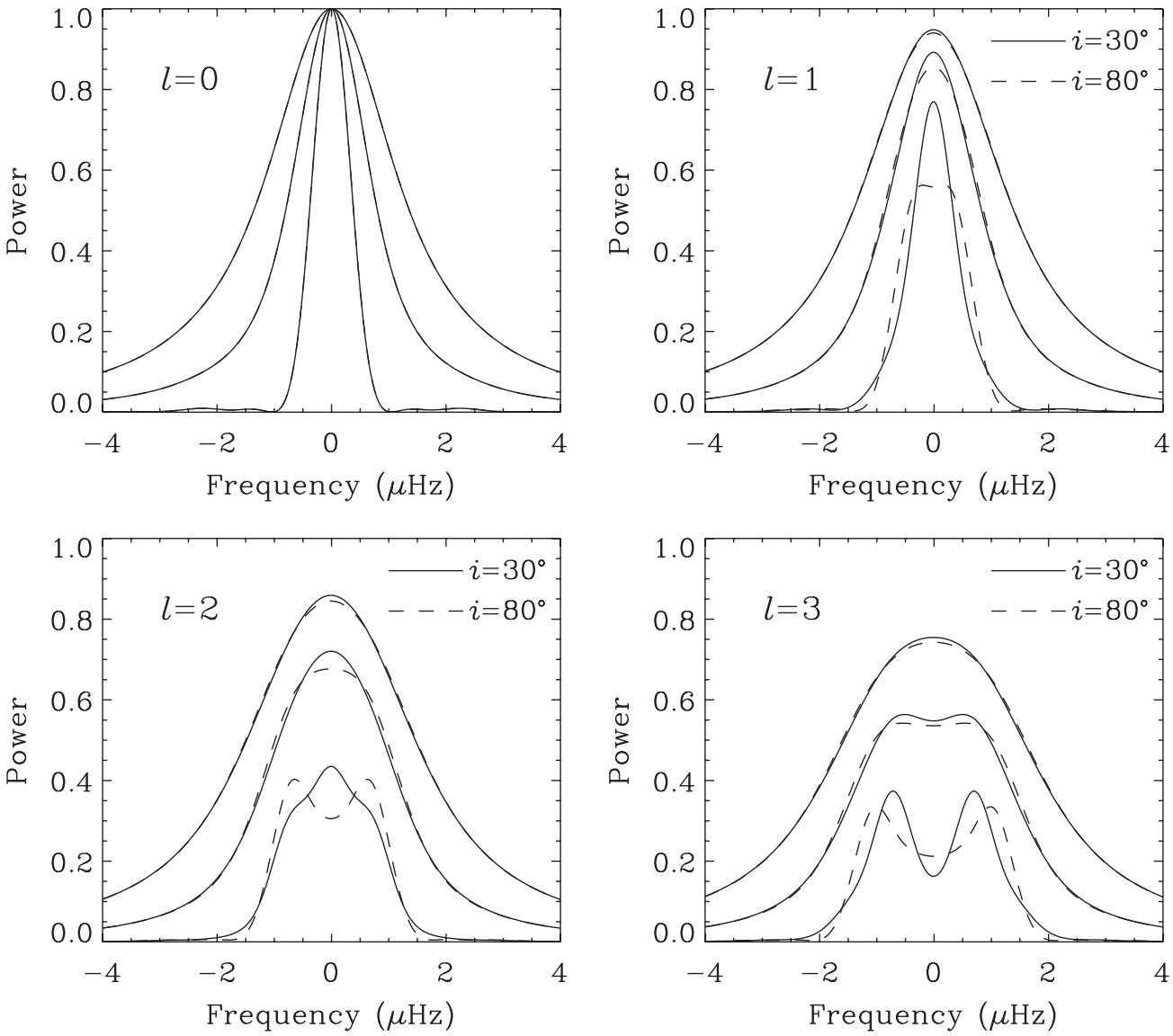

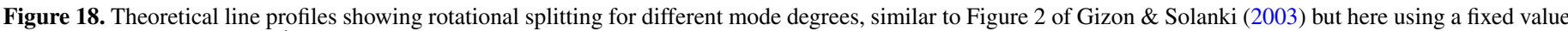

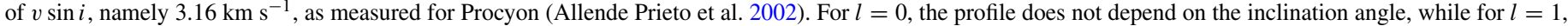

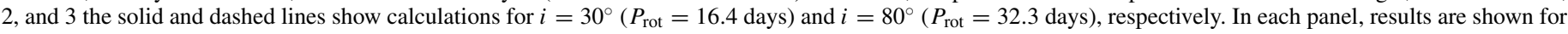

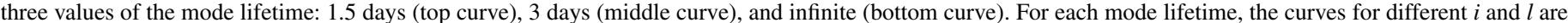
all normalized to have the same area.

We see from Figure 18 that for a fixed $v \sin i$, the width of the profile stays roughly constant as a function of inclination. If the rotation axis of the star happens to be in the plane of the sky $\left(i=90^{\circ}\right)$, then the rotation period is too low to produce a measurable splitting. At the other extreme, if the inclination is small (so that the rotation is close to pole-on), then the rotational splitting will be large but most of the power will be in the central peak $(m=0)$. Either way, once the profile has been broadened by the mode lifetime, the splitting will be unobservable.

We conclude that for realistic values of the mode lifetime, our observations are not long enough to detect rotational splitting in Procyon. The line profiles are broadened by rotation, but it is not possible to disentangle the rotation rate from the inclination angle. Rotational splitting is not measurable in Procyon, except perhaps with an extremely long data set. The detection of rotational splitting requires choosing a star with a larger $v \sin i$ or a longer mode lifetime, or both.

\section{APPENDIX B \\ RELATING RIDGE CENTROIDS TO MODE FREQUENCIES}

As discussed in Section 5, the frequencies of the ridge centroids are useful for asteroseismology in cases where it is difficult to resolve the ridges into their component modes. In this appendix, we relate the frequencies of the ridge centroids to those of the underlying modes, which allows us to express the small separation of the ridges (Equation (10)) in terms of the conventional small separations $\left(\delta v_{01}, \delta v_{02}\right.$, and $\left.\delta v_{13}\right)$. These relationships will allow the observations to be compared with theoretical models.

The ridge centroids depend on the relative contributions of modes with $l=0,1,2$, and 3 . The power in the even ridge is approximately equally divided between $l=0$ and $l=2$, while the odd ridge is dominated by $l=1$ but with some contribution from $l=3$. The exact ratios depend on the observing method, as discussed by Kjeldsen et al. (2008a). For velocity measurements, such as those presented in this paper for Procyon, the amplitude ratios given by Kjeldsen et al. (2008a, their Table 1) yield the following expressions for the centroids in power:

$$
\begin{aligned}
& v_{n, \text { even }}^{\mathrm{vel}}=0.49 v_{n, 0}+0.51 v_{n-1,2}, \\
& v_{n, \text { odd }}^{\mathrm{vel}}=0.89 v_{n, 1}+0.11 v_{n-1,3},
\end{aligned}
$$

where the superscript indicates these apply to velocity measurements.

For photometric measurements, such as those currently being obtained with the CoRoT and Kepler Missions, the relative contributions from the various $l$ values are different. Table 1 of Kjeldsen et al. (2008a) gives response factors for intensity measurements in the three VIRGO passbands, namely, 402, 500 , and $862 \mathrm{~nm}$. For CoRoT and Kepler, it is appropriate to use a central wavelength of $650 \mathrm{~nm}$. Using the same method as Kjeldsen et al. (2008a), we find the ratios (in amplitude) for this case to be $S_{0}: S_{1}: S_{2}: S_{3}=1.00: 1.23: 0.71: 0.14$. The 
ridge centroids measured from such data would then be

$$
\begin{aligned}
& v_{n, \text { even }}^{650}=0.66 v_{n, 0}+0.34 v_{n-1,2}, \\
& v_{n, \text { odd }}^{650}=0.99 v_{n, 1}+0.01 v_{n-1,3} .
\end{aligned}
$$

We can express the new small separation of the ridge centroids (Equation (10)) in terms of the conventional ones. For velocity, we have

$$
\delta v_{\mathrm{even}, \text { odd }}^{\mathrm{vel}}=\delta v_{01}-0.51 \delta v_{02}+0.11 \delta v_{13}
$$

and for photometry, we have

$$
\delta v_{\text {even,odd }}^{650}=\delta v_{01}-0.34 \delta v_{02}+0.01 \delta v_{13}
$$

Finally, we can express these in terms of $D_{0}$ under the assumption that the asymptotic relation (Equation (1)) holds exactly, although in fact this is not likely to be the case:

$$
\delta v_{\text {even,odd }}^{\mathrm{vel}}=0.04 D_{0}
$$

and

$$
\delta v_{\text {even,odd }}^{650}=0.06 D_{0}
$$

\section{REFERENCES}

Aerts, C., Christensen-Dalsgaard, J., Cunha, M., \& Kurtz, D. W. 2008, Sol. Phys., 251, 3

Aizenman, M., Smeyers, P., \& Weigert, A. 1977, A\&A, 58, 41

Allende Prieto, C., Asplund, M., López, R. J. G., \& Lambert, D. L. 2002, ApJ, 567,544

Anderson, E. R., Duvall, T. L., \& Jefferies, S. M. 1990, ApJ, 364, 699

Appourchaux, T., et al. 2008, A\&A, 488, 705

Arentoft, T., Kjeldsen, H., \& Bedding, T. R. 2010, in ASP Conf. Ser. 416, GONG 2008/SOHO XXI Meeting on Solar-Stellar Dynamos as Revealed by Helioand Asteroseismology, ed. M. Dikpati et al. (San Francisco, CA: ASP), in press (arXiv:0901.3632)

Arentoft, T., et al. 2008, ApJ, 687, 1180 (Paper I)

Aufdenberg, J. P., Ludwig, H.-G., \& Kervella, P. 2005, ApJ, 633, 424

Ballot, J., García, R. A., \& Lambert, P. 2006, MNRAS, 369, 1281

Ballot, J., Turck-Chièze, S., \& García, R. A. 2004, A\&A, 423, 1051

Barban, C., Michel, E., Martić, M., Schmitt, J., Lebrun, J. C., Baglin, A., \& Bertaux, J. L. 1999, A\&A, 350, 617

Barban, C., et al. 2009, A\&A, 506, 51

Baudin, F., Gabriel, A., Gibert, D., Pallé, P. L., \& Régulo, C. 1996, A\&A, 311, 1024

Bedding, T. R., \& Kjeldsen, H. 2003, PASA, 20, 203

Bedding, T. R., \& Kjeldsen, H. 2007, in AIP Conf. Proc. 948, Unsolved Problems in Stellar Physics: A Conference in Honour of Douglas Gough, ed. R. J. Stancliffe et al. (Melville, NY: AIP), 117

Bedding, T. R., \& Kjeldsen, H. 2010, Commun. Asteroseismol., 161, 3

Bedding, T. R., Kjeldsen, H., Butler, R. P., McCarthy, C., Marcy, G. W., O’Toole,

S. J., Tinney, C. G., \& Wright, J. T. 2004, ApJ, 614, 380

Bedding, T. R., et al. 2007, ApJ, 663, 1315

Bedding, T. R., et al. 2010, ApJ, in press (arXiv:1001.0229)

Benomar, O., Appourchaux, T., \& Baudin, F. 2009a, A\&A, 506, 15

Benomar, O., et al. 2009b, A\&A, 507, L13

Bonanno, A., Küker, M., \& Paternò, L. 2007, A\&A, 462, 1031

Broomhall, A.-M., Chaplin, W. J., Davies, G. R., Elsworth, Y., Fletcher, S. T., Hale, S. J., Miller, B., \& New, R. 2009, MNRAS, 396, L100

Brown, T. M., \& Gilliland, R. L. 1994, ARA\&A, 32, 37

Carrier, F., et al. 2007, A\&A, 470, 1059

Carrier, F., et al. 2010, A\&A, 55, A73

Chaboyer, B., Demarque, P., \& Guenther, D. B. 1999, ApJ, 525, L41

Chang, H.-Y., \& Gough, D. O. 1998, Sol. Phys., 181, 251

Chaplin, W. J., Elsworth, Y., Isaak, G. R., McLeod, C. P., Miller, B. A., \& New, R. 1997, MNRAS, 288, 623

Chaplin, W. J., Houdek, G., Elsworth, Y., Gough, D. O., Isaak, G. R., \& New, R. 2005, MNRAS, 360, 859

Chaplin, W. J., Houdek, G., Karoff, C., Elsworth, Y., \& New, R. 2009, A\&A, 500, L21
Christensen-Dalsgaard, J. 2004, Sol. Phys., 220, 137

Christensen-Dalsgaard, J., Däppen, W., \& Lebreton, Y. 1988, Nature, 336, 634

Christensen-Dalsgaard, J., \& Houdek, G. 2009, Ap\&SS, in press (arXiv:0911.4629)

Deheuvels, S., et al. 2010, A\&A, submitted

Di Mauro, M. P., \& Christensen-Dalsgaard, J. 2001, in IAU Symp. 203, Recent Insights into the Physics of the Sun and Heliosphere: Highlights from SOHO and Other Space Missions, ed. P. Brekke, B. Fleck, \& J. B. Gurman (San Francisco, CA: ASP), 94

Dziembowski, W. A., Paternó, L., \& Ventura, R. 1988, A\&A, 200, 213

Eggenberger, P., Carrier, F., \& Bouchy, F. 2005, New Astron., 10, 195

Eggenberger, P., Carrier, F., Bouchy, F., \& Blecha, A. 2004, A\&A, 422, 247

Fletcher, S. T., Chaplin, W. J., Elsworth, Y., Schou, J., \& Buzasi, D. 2006, MNRAS, 371, 935

Frandsen, S., Jones, A., Kjeldsen, H., Viskum, M., Hjorth, J., Andersen, N. H., \& Thomsen, B. 1995, A\&A, 301, 123

García, R. A., et al. 2009, A\&A, 506, 41

Gatewood, G., \& Han, I. 2006, AJ, 131, 1015

Gilliland, R. L., et al. 1993, AJ, 106, 2441

Gilliland, R. L., et al. 2010, PASP, 122, 131

Girard, T. M., et al. 2000, AJ, 119, 2428

Gizon, L., \& Solanki, S. K. 2003, ApJ, 589, 1009

Gough, D. O. 1986, in Hydrodynamic and Magnetodynamic Problems in the Sun and Stars, ed. Y. Osaki (Tokyo: Univ. Tokyo Press), 117

Gough, D. O. 1990, in Lecture Notes in Physics, Vol. 367, Progress of Seismology of the Sun and Stars, ed. Y. Osaki \& H. Shibahashi (Berlin: Springer), 283

Grec, G., Fossat, E., \& Pomerantz, M. A. 1983, Sol. Phys., 82, 55

Gregory, P. C. 2005, Bayesian Logical Data Analysis for the Physical Sciences (Cambridge: Cambridge Univ. Press)

Gruberbauer, M., Kallinger, T., Weiss, W. W., \& Guenther, D. B. 2009, A\&A, 506, 1043

Grundahl, F., Christensen-Dalsgaard, J., Arentoft, T., Frandsen, S., Kjeldsen, H., Jørgensen, U. G., \& Kjaergaard, P. 2008, Commun. Asteroseismol., 157, 273

Guenther, D. B., \& Demarque, P. 1993, ApJ, 405, 298

Guenther, D. B., et al. 2008, ApJ, 687, 1448

Högbom, J. A., \& Brouw, W. N. 1974, A\&A, 33, 289

Houdek, G., \& Gough, D. O. 2007, MNRAS, 375, 861

Jeffreys, H. 1961, Theory of Probability (3rd ed.; New York: Oxford Univ. Press)

Kallinger, T., Gruberbauer, M., Guenther, D. B., Fossati, L., \& Weiss, W. W. 2010, A\&A, 510, A106

Karoff, C. 2007, MNRAS, 381, 1001

Kervella, P., Thévenin, F., Morel, P., Berthomieu, G., Bordé, P., \& Provost, J. 2004, A\&A, 413, 251

Kjeldsen, H., Bedding, T. R., \& Christensen-Dalsgaard, J. 2008b, ApJ, 683, L175

Kjeldsen, H., Bedding, T. R., Viskum, M., \& Frandsen, S. 1995, AJ, 109, 1313

Kjeldsen, H., et al. 2005, ApJ, 635, 1281

Kjeldsen, H., et al. 2008a, ApJ, 682, 1370

Leccia, S., Kjeldsen, H., Bonanno, A., Claudi, R. U., Ventura, R., \& Paternò, L. 2007, A\&A, 464, 1059

Li, L. H., Robinson, F. J., Demarque, P., Sofia, S., \& Guenther, D. B. 2002, ApJ, 567,1192

Liddle, A. R. 2009, Annu. Rev. Nucl. Part. Sci., 59, 95

Martić, M., Lebrun, J.-C., Appourchaux, T., \& Korzennik, S. G. 2004, A\&A, 418, 295

Martić, M., et al. 1999, A\&A, 351, 993

Mathur, S., et al. 2010, A\&A, submitted

Michel, E., et al. 2008, Science, 322, 558

Mosser, B., \& Appourchaux, T. 2009, A\&A, 508, 877

Mosser, B., Appourchaux, T., Catala, C., Buey, J., \& SIAMOIS team 2008, J. Phys. Conf. Ser., 118, 012042

Mosser, B., Maillard, J. P., Mékarnia, D., \& Gay, J. 1998, A\&A, 340, 457

Osaki, J. 1975, PASJ, 27, 237

Provost, J., Berthomieu, G., Martić, M., \& Morel, P. 2006, A\&A, 460, 759

Régulo, C., \& Roca Cortés, T. 2005, A\&A, 444, L5

Rosenthal, C. S., Christensen-Dalsgaard, J., Nordlund, Å., Stein, R. F., \& Trampedach, R. 1999, A\&A, 351, 689

Roxburgh, I. W. 2009, A\&A, 506, 435

Roxburgh, I. W., \& Vorontsov, S. V. 2006, MNRAS, 369, 1491

Tassoul, M. 1980, ApJS, 43, 469

Toutain, T., \& Fröhlich, C. 1992, A\&A, 257, 287

van Leeuwen, F. 2007, Hipparcos, the New Reduction of the Raw Data (Dordrecht: Springer) 